\title{
Importancia del tiempo efectivo de instrucción en el desempeño académico: resultados de una aproximación mixta en Colombia
}

\author{
Recibido: julio, 03 de 2018 - Aprobado: agosto, 23 de 2018
}

Doi: http://dx.doi.org/10.12804/revistas.urosario.edu.co/economia/a.7763

Alexander Villegas Mendoza*

\section{Resumen}

Se implementaron variables instrumentales para estimar el efecto del tiempo efectivo de instrucción en el desempeño de los estudiantes colombianos en la prueba Saber 11. Se encontró que aumentar en una desviación estándar el tiempo efectivo de instrucción incrementa el puntaje en los componentes de Matemáticas en 0,43 desviaciones y Lenguaje en 0,16 , respectivamente. Una aproximación cualitativa en el departamento del Atlántico sugiere que la ventilación de las aulas, la temperatura, la disciplina, los desplazamientos hacia la escuela, las actividades ajenas a las clases y las interrupciones de los padres son los factores que más afectan el desarrollo de las actividades académicas.

Palabras clave: tiempo efectivo de instrucción, desempeño académico, Saber 11, variables instrumentales, métodos mixtos.

Clasificación JEL: I21, I28, Y80.

* Profesor catedrático Departamento de Economía y asistente de proyectos del Observatorio de Educación del Caribe Colombiano, Universidad del Norte.

Correspondencia: Km. 5 Vía Puerto Colombia, bloque B segundo piso. Departamento de Economía, Universidad del Norte. Correo electrónico: villegas@uninorte.edu. co y a.villegas177@uniandes.edu.co

Para citar este artículo: Villegas Mendoza, A. (2019). Importancia del tiempo efectivo de instrucción en el desempeño académico: resultados de una aproximación mixta en Colombia. Revista de Economía del Rosario, 22(1), 5-51. Dor: http:/ /dx.doi.org/10.12804/revistas. urosario.edu.co/economia/a.7763 


\title{
Importance of Effective Instructional Time in Academic Achievement: Results of a Mixed Approach in Colombia
}

\begin{abstract}
Instrumental variables were implemented to estimate the consequences of effective instructional time on the performance of Colombian students in the Saber 11 examination. When increasing in a standard deviation, the effective instruction time increments the scores by 0.43 and 0.16 in Math and Language components of this test, respectively. A qualitative approach in the Atlantico department suggests that factors such as classroom ventilation, temperature, discipline, trips to school, activities different to classes, and interruptions from parents are which most affect the development of academic activities.
\end{abstract}

Keywords: Effective instructional time, academic achievement, Saber 11, instrumentals variables, mixed methods.

JEL Classification: I21, I28, Y80.

\section{Importância do tempo efetivo de instrução no desempenho acadêmico: resultados de uma aproximação mista na Colômbia}

\section{Resumo}

Se implementaram variáveis instrumentais para estimar o efeito do tempo efetivo de instrução no desempenho dos estudantes colombianos na prova Saber 11. Se encontrou que aumentar em um desvio-padrão o tempo efetivo de instrução incrementa em 0.43 e 0.16 desvios-padrões a pontuação nos componentes de Matemáticas e Linguagem, respetivamente. Uma aproximação qualitativa no departamento do Atlântico sugere que a ventilação das aulas, a temperatura, a disciplina, os deslocamentos para a escola, atividades alheias às aulas e as interrupções de pais são os que mais afetam o desenvolvimento das atividades acadêmicas.

Palavras-chave: tempo efetivo de instrução, desempenho acadêmico, Saber 11, variáveis instrumentais, métodos mistos.

Classificação JEL: I21, I28, Y80. 


\section{Antecedentes y justificación}

En un reciente y pionero trabajo para Latinoamérica, Bruns y Luque (2014) exponen que el bajo rendimiento de los estudiantes en las pruebas estandarizadas que se realizan en la región se encuentra relacionado con el porcentaje de tiempo de clase que los profesores dedican a las actividades académicas (denominado en la presente investigación como tiempo efectivo de instrucción ${ }^{1}$ ), entre las que se incluyen la lectura en voz alta del docente o por parte de uno o varios de los estudiantes; actividades de exposición o demostración; espacios para preguntas y respuestas o discusiones; práctica y ejercicios; supervisar trabajo individual de los estudiantes, y copia de texto del tablero por parte de estos últimos (World Bank Group, 2015). En particular, estos autores calculan que los docentes colombianos dedican cerca del $65 \%$ del tiempo de clase a las actividades académicas -el mayor porcentaje entre los países observados-, veinte puntos porcentuales menos que el marco de referencia establecido por el sistema educativo estadounidense y lo que equivale a perder un día de clase a la semana. ${ }^{2}$ Sin embargo, los autores no establecen una relación causal entre el desempeño de los estudiantes y el tiempo efectivo de enseñanza, limitándose a presentar una correlación entre ambas variables (Bruns \& Luque, 2014), aspecto que el presente trabajo abordó a partir de la misma base de datos de observaciones en el aula empleada por estos investigadores.

Teniendo en cuenta el contexto educativo actual del país -marcado por los bajos resultados obtenidos en la prueba PISA ${ }^{3}$ en 2012 (ICFES, 2013), con avances en cobertura, pero donde la calidad educativa sigue siendo el punto clave de política a resolver (Barrera, Maldonado \& Rodríguez, 2012) y en el que la distribución de la educación es desigual a lo largo de la geografía nacional (Burgos, 2011; Kairuz, Correa, Durán, Godoy \& Perilla, 2008)-, el gobierno del expresidente Juan Manuel Santos durante su segundo periodo 2014-2018 se propuso hacer del país la nación más educada de Latinoamé-

1 Se usará esta definición para diferenciarlo del instructional time, utilizado en la literatura para referirse a la extensión de la jornada académica diaria (medida en horas de clases) o el año escolar (medido en días o semanas).

2 Adicionalmente, los resultados de estos autores permiten observar que los docentes del país dedican aproximadamente un $25 \%$ del tiempo de clases a actividades relacionadas con la gestión de la clase, estas incluyen llamar a lista, acciones encaminadas a mantener la disciplina del curso, revisar trabajos, brindar instrucciones o manejar el aula, mientras que el restante $10 \%$ del tiempo los docentes realizan actividades ajenas al desarrollo de la clase o su manejo, tales como ausentarse del salón e interactuar socialmente con estudiantes o personas ajenas a la clase.

3 Programa Internacional de Evaluación de Estudiantes, por su sigla en inglés. 
rica para 2025 (DNP, 2015) y desarrollar un conjunto de acciones que buscan mejorar la calidad educativa, entre las que se encuentran la implementación gradual de la jornada única en las instituciones educativas oficiales del país, la cual tiene como objetivo aumentar el tiempo que los estudiantes pasan en las escuelas (DNP, 2015), y la estrategia denominada "hacia la excelencia docente" que incluye acciones que "[...] promuevan el mejoramiento del uso del tiempo en el aula por parte de los docentes [...]" (DNP, 2015, p. 88).

Sin embargo, en la mayoría de países hay poca información sobre el uso efectivo del tiempo de clase y su relación con el desempeño académico de los estudiantes (Abadzi, 2009) que respalde decisiones de política pública como la expuesta anteriormente. De esta forma, en este trabajo se estimó causalmente esta relación, midiendo el desempeño académico por los resultados en la prueba Saber 11 de 2011. En este sentido, se adoptó una aproximación mixta con un estudio de caso en dos municipios del departamento del Atlántico (Colombia), de García, Fernández y Weiss (2013) y Palacios, Sánchez y Córdoba (2015), para obtener mayor información sobre elementos que pudiesen afectar el uso del tiempo en las aulas y los procesos subyacentes en la relación estudiada.

Los resultados de esta investigación aportan evidencia de que el tiempo efectivo de instrucción es un elemento relevante en el desempeño académico de los estudiantes, y que tiene mayor incidencia sobre los resultados en Matemáticas. Así, un aumento del $10 \%$ del tiempo efectivo de instrucción tiene incrementos en Matemáticas de 0,43 desviaciones estándar y en lenguaje de 0,16, respectivamente. En términos comparativos, las estimaciones de Bellei (2009) sobre el efecto de la jornada única en Chile para estas dos asignaturas se ubican entre 0,05-0,07 y 0,00-0,12 desviaciones estándar. Así mismo, intervenciones para reducir el ausentismo docente en India y Kenia han mostrado efectos de 0,21 desviaciones estándar en Lenguaje (Duflo, Hanna \& Ryan, 2012) y de 0,16-0,21 desviaciones estándar en matemáticas (Duflo, Dupas \& Kremer, 2015; Duflo et al., 2012).

Además, la aproximación cualitativa permitió explorar que elementos como la disciplina, la ventilación de los cursos, los desplazamientos hacia la escuela, los horarios de atención a los padres de familia y la realización de eventos ajenos a las clases son, según los docentes participantes, los que más afectan al tiempo efectivo de instrucción. Más aún, los docentes sostienen que el tiempo del que disponen para desarrollar los contenidos de sus asignaturas es insuficiente, por lo que corregir los factores que reducen el tiempo efectivo de instrucción contribuiría a mejorar el desempeño de los estudiantes.

Este documento contiene cinco secciones, además de esta introducción, que es la primera. En la segunda se presenta una revisión de la literatura y 
en la tercera se aborda la metodología de las aproximaciones cuantitativas y cualitativas. En la cuarta se exponen los resultados de ambos enfoques y en la quinta se presentan las conclusiones y las recomendaciones de política pública.

\section{Revisión de literatura}

Al explicar las diferencias en el desempeño académico de los estudiantes, se ha encontrado relación entre los factores personales, los familiares y la comunidad. De la misma forma, en cuanto al colegio se refiere, el tamaño de la clase (Mckee, Rivkin \& Sims, 2010),el bullying o matoneo (van der Werf, 2014) y el gasto por estudiante (Mina, 2004) son algunas de las variables que han cumplido tal proposito. El presente trabajo se concentra en el análisis del uso del tiempo efectivo de instrucción por los docentes, un elemento asociado a las prácticas pedagógicas del aula, ${ }^{4}$ sumándolo al cuerpo de literatura que ha buscado cuantificar la relación entre la calidad de los profesores y el logro académico de los estudiantes (García, Maldonado, Perry, Rodríguez \& Saavedra, 2014a y 2014b; Hanushek, 1971; Rivkin, Hanushek \& Kain, 2005; Woessmann, 2016). Esa labor no es sencilla, si se considera que diferentes estudios no han encontrado relación de este último con variables de los docentes comúnmente capturadas por las bases de datos tradicionales (Hanushek, 2016, 2011 y 1971; Rivkin et al., 2005).

Puede decirse que la literatura sobre el tiempo de clase y el desempeño académico se ha agrupado en factores como (i) la duración de la jornada académica, (ii) el ausentismo de los docentes y (iii) el uso efectivo del tiempo de instrucción en clase por parte de los maestros (porcentaje del tiempo de clase dedicado a actividades académicas). Respecto al primer enfoque, se ha encontrado evidencia de un efecto positivo de la extensión de la jornada académica en el desempeño académico en diferentes investigaciones; por ejemplo, Rivkin y Schiman (2013) y Lavy (2010) utilizan datos panel con efectos fijos de estudiantes y escuela para verificar tal relación en los resultados en las pruebas PISA de los años 2006 y 2009. Así mismo, Lavy (2012) explota un

4 UNESCO (2015) aporta un reciente estudio sobre prácticas docentes en el aula en diferentes países de Latinoamérica (incluido Colombia). En este documento se expone una relación positiva entre la asistencia y puntualidad de los docentes sobre el desempeño académico de los estudiantes. Lo anterior, también ocurre para los alumnos que poseen cuadernos y libros. De forma paralela, elaboran un índice asociado a prácticas docentes, el cual no muestra relación con el desempeño de los estudiantes de sexto grado en Matemáticas y Lenguaje en Colombia. Otros trabajos interesantes en la materia son los elaborados por Kane, Taylor, Tayler y Wooten (2011) y Méndez (2013). 
cambio exógeno en la asignación del presupuesto escolar en Israel ${ }^{5}$ y obtiene resultados en la misma dirección en pruebas estatales de dicho país. Por otra parte, Goodman (2014) estima mediante efectos fijos y variables instrumentales que las ausencias por mal clima disminuyen el logro en Matemáticas de los estudiantes del estado de Massachusetts (Estados Unidos).

De forma similar, García et al. (2013) estiman una reducción de la probabilidad de deserción y tasas de repitencia para los estudiantes que asisten a jornada completa, mientras que Hincapié (2016) emplea datos panel para los años 2002, 2005 y 2009, y encuentra un efecto positivo en el desempeño de los estudiantes en las pruebas Saber 5 y Saber 9 que esta relacionado con las jornadas educativas más extensas, y Bellei (2009) estima un efecto positivo en el desempeño académico de los estudiantes chilenos de jornada completa en Matemáticas y Lenguaje. Bonilla (2011) argumenta que los estudiantes en jornada completa obtienen mejores puntajes que los de otras, especialmente al compararlos con aquellos que estudian en la jornada de la tarde; este último autor emplea como variable instrumental la oferta de colegios de jornada única en el municipio para resolver el problema de endogeneidad.

Por su parte, las consecuencias del ausentismo docente y los mecanismos para reducirlo ${ }^{6}$ han sido estudiadas ampliamente por autores como Esther Duflo. Esta autora y otros académicos emplearon en un experimento aleatorio el monitoreo diario con cámaras y un incentivo salarial para intentar reducir este problema en una provincia en la India (Duflo et al., 2012). Sus resultados mostraron una disminución de 21 puntos porcentuales en la tasa de ausencias de los profesores y un aumento de 0,21 y 0,16 desviaciones estándar en los resultados de los estudiantes en Lenguaje y Matemáticas (Duflo et al., 2012). Para este mismo país, Muralidharan, Das, Holla y Mohpal (2017) muestran que una intervención basada en el monitoreo (específicamente, contratar más supervisores) es 12,8 veces más costo efectivo que contratar más docentes. Así mismo, la relación negativa entre las ausencias de los docentes y los resultados en matemáticas son consistentes con los del estudio citado anteriormente y otros elaborados en esta línea (Muralidharan et al., 2017).

5 El autor explota este cambio exógeno y su efecto sobre el presupuesto de enseñanza por clase, la duración de la semana académica y en el tiempo de instrucción en las escuelas de este país. Acto seguido, examina su efecto en los puntajes de los estudiantes en pruebas de Matemáticas, Ciencias e Inglés. Lo anterior, mediante la diferencia de la variable de resultado de una escuela antes y después del cambio de legislación, además de emplear diferencias en diferencias.

6 Banerjee y Duflo (2006) ofrecen un recuento de experiencias sobre el reto de resolver el ausentismo docente. 
En otro estudio, Duflo et al. (2015) evaluaron un programa en Kenia que buscaba brindarle a las escuelas la oportunidad de contratar a un docente y remunerarlo de acuerdo con su rendimiento; mediante esta estrategia y el empoderamiento de los padres de familia se aumentó en 12 puntos porcentuales la probabilidad de encontrar a los docentes enseñando y en 0,21 desviaciones estándar los resultados en matemáticas de los estudiantes (Duflo et al., 2015). Para el caso de Colombia, se han encontrado niveles menores de ausentismo y mayor puntualidad en los docentes de colegios de alto desempeño en comparación con los de bajo y que estas diferencias son estadísticamente significativas (García et al., 2014d).

Respecto al uso efectivo del tiempo de clase, Abadzi (2009) afirma que en los países en desarrollo solo una porción de las horas establecidas de clase es implementada de forma cierta; en su trabajo expone que se pierde tiempo de clase ya sea por la apertura más tarde de las escuelas o su cierre más temprano en comparación con lo establecido en el calendario oficial, el ausentismo de los profesores o sus llegadas tarde, además del desperdicio del tiempo en la clase misma. En esta línea, Venäläinen (2008) utiliza el Stallings Classroom Snapshot ${ }^{7}$ y calcula que en las escuelas observadas los docentes dedicaban en promedio un $70,9 \%$ del tiempo de clase a actividades académicas. Por su parte, los estudiantes estaban atentos a la clase en el 65,7\% del tiempo (Venäläinen, 2008).

De igual modo, Bruns y Luque (2014) analizan, en un estudio pionero para Latinoamérica, la utilización del tiempo de instrucción por los docentes de siete países de esta región. Esta investigación implementó observaciones en más de 15.000 aulas en 3.000 instituciones educativas de la región a través del protocolo Stallings Classroom Snapshot (Bruns \& Luque, 2014). La principal conclusión que se extrae de este trabajo es que el bajo rendimiento de los estudiantes de la región en las pruebas estandarizadas de cada país se relaciona con el escaso porcentaje del tiempo de clase que sus profesores dedican a las actividades de enseñanza-aprendizaje (Bruns \& Luque, 2014)-denominado en la presente investigación como tiempo efectivo de instrucción-; entre estas se incluyen la lectura en voz alta del docente o por parte de uno o varios de los

7 Este método consiste en la captura de diez instantáneas de 15 segundos, cada una distribuida a lo largo de la clase, en las cuales una persona entrenada realiza una observación de $360^{\circ}$ del aula. En cada una se analiza el uso del tiempo de instrucción, el uso de materiales, prácticas pedagógicas y la habilidad del docente de mantener al curso involucrado con la clase. Este método genera información cuantitativa robusta sobre la interacción de estudiantes y profesores en el aula. Para un resumen del método ver Bruns y Luque (2014, pp. 100-105), y para una explicación más detallada del mismo, así como sobre la forma en que se codifican las observaciones ver World Bank Group (2015). 
estudiantes, las actividades de exposición o demostración, los espacios para preguntas y respuestas o discusiones, las prácticas y ejercicios, supervisar el trabajo individual de los estudiantes y la copia de texto del tablero por parte de estos últimos (World Bank Group, 2015).

El trabajo desarrollado por Bruns y Luque (2014) no establece una relación causal entre las variables y se limita a una correlación entre las mismas. La presente investigación pretende resolver este vacío a partir de la misma base de datos de observaciones en el aula empleada por estos investigadores, pero recurriendo a una estrategia causal. Por consiguiente, aporta evidencia en un área poco explorada, la relación entre el tiempo efectivo de instrucción y el desempeño académico de los estudiantes enfocándose en el caso de Colombia (Abadzi, 2009).

\section{Metodología}

En este apartado se exponen los métodos y técnicas elegidas para cumplir los objetivos de esta investigación; se relaciona la metodología econométrica empleada, las técnicas cualitativas de captura de información aplicadas, los criterios de selección de muestra y la herramienta de análisis.

\subsection{Aproximación cuantitativa}

El punto común de las investigaciones en economía de la educación es la solución de los problemas de endogeneidad de la variable de interés o su relación con otros determinantes (Lavy, 2010). El ejemplo más común sucede al evaluar el impacto causal de una variable específica en medidas de calidad de la educación de los estudiantes a través de estimaciones econométricas. En este sentido, una estimación de la relación entre el tiempo efectivo de instrucción y el desempeño académico por Mínimos Cuadrados Ordinarios (мсо) produciría resultados inconsistentes, debido a elementos como las decisiones de localización y escolarización que acuerdan las familias (Meghir \& Rivkin, 2011), tales como contratar clases adicionales para complementar el tiempo de instrucción recibido en las escuelas (Rivkin \& Schiman, 2013), y la elección de las mismas.

Otros elementos a tener en cuenta en este caso son el emparejamiento no aleatorio entre docentes y estudiantes; el proceso político y legal que determina las finanzas escolares, y por ende los recursos de que disponen los alumnos; y la maximización de utilidad de los profesores y demás personal al decidir las escuelas en las que trabajan (Meghir \& Rivkin, 2011). En Colombia, los postulantes con mejores resultados en las pruebas de acceso a 
la carrera docente tienen la facultad de elegir primero entre las plazas disponibles (Resolución 0207 de 2010). Estos se concentran en municipios ubicados en la región Andina colombiana y en aquellos con menor pobreza, ataques terroristas y desplazamiento (García et al., 2014c). Así mismo, es causal de endogeneidad la asignación no aleatoria de estudiantes a cursos específicos por características como la edad (Lavy, 2012).

Por consiguiente, se empleó una aproximación de variables instrumentales, como la utilizada por Bonilla (2011), quien instrumenta con la oferta de colegios de jornada única en el municipio para analizar el efecto de escuelas de este tipo en el desempeño académico; van der Werf (2014), quien analiza el efecto del matoneo en el desempeño académico mediante la instrumentación con la desviación estándar de la edad de los estudiantes en la escuela, y Goodman (2014), que instrumenta con la intensidad de las nevadas para estudiar el efecto de las ausencias escolares en la misma variable de interés de los trabajos anteriores.

Esta metodología permite obtener estimadores insesgados y consistentes mediante la utilización de una variable instrumental (Rosales, Perdomo, Morales \& Urrego, 2013). Esta última se relaciona con la explicativa que causa el problema de endogeneidad (en este caso, el tiempo efectivo de instrucción), pero es independiente del término de error del modelo para garantizar la consistencia del estimador (Cameron \& Trivedi, 2005; Rosales et al., 2013). En vista de lo anterior, se emplearon Mínimos Cuadrados en Dos Etapas (MC2E), mediante la estimación de una regresión como la descrita por la ecuación 1 entre los instrumentos (edad promedio de docentes $s_{e, m}$ y edad promedio de docentes ${ }_{e, m}^{2}$ ) y la variable endógena (Tiempo efectivo de instrucción ${ }_{e, m}$ ) para obtener la porción exógena de la misma. Esta especificación incluye también a las demás variables exógenas del modelo.

Tiempo efectivo de instrucción $n_{e, m}$

$$
\begin{aligned}
& =\gamma_{0}+\gamma_{1} \text { edad promedio de docentes } s_{\mathrm{e}, \mathrm{m}} \\
& +\gamma_{2} \text { edad promedio de docentes }_{\mathrm{e}, \mathrm{m}}+\gamma_{3} X_{\mathrm{i}, \mathrm{e}, \mathrm{m}}+\gamma_{4} S_{\mathrm{e}, \mathrm{m}} \\
& +\gamma_{m}+v_{\mathrm{e}, \mathrm{m}}
\end{aligned}
$$

Se usaron estos instrumentos debido a que cumplen con la condición de relevancia, tal y como puede observarse en los resultados de la primera etapa, en la sección de Resultados (Wooldridge, 2002). La intuición detrás de ello es que la edad del docente puede repercutir en un mejor manejo del desarrollo de la clase y disminuir así las interrupciones; lo anterior, especulando, puede aumentar el tiempo efectivo de instrucción en las aulas. Acto seguido, debido a que no hay una prueba estadística que valide la exogeneidad del instrumento 
(Pokroprek, 2016), se recurrió a bibliografía y se argumentó el cumplimiento de la restricción de exclusión. En este sentido, se consideró la edad promedio de los docentes como un elemento aleatorio que solo depende de sus propias fechas de nacimiento y no de las decisiones de padres o estudiantes, es decir, se asume que la edad no afecta la capacidad de los docentes de transmitir conocimiento a los estudiantes ni el desempeño académico de estos.

La defensa del cumplimiento de la restricción de exclusión por parte de los instrumentos siempre es un asunto complejo y no exento de críticas; no obstante, se ofrecen como argumentos a favor el hecho de que diferentes estudios (Rivkin et al. 2005; Hanushek, 2016) han encontrado poca relación entre las características comúnmente medibles de los docentes y el rendimiento académico, mientras que otros estiman poca o nula relación con la edad (García et al., 2014b; Méndez, 2013). Por otra parte, puede suponerse una relación estrecha entre la edad del docente y su experiencia, así como de esta con el rendimiento académico de los estudiantes; sin embargo, no hay evidencia concluyente de que estas dos últimas variables se relacionen causalmente (Rivkin et al. 2005; Glewwe, Hanushek, Humpage \& Ravina, 2013; Hanushek, 2011, 1971) y la relación específica entre las mismas es dificil de interpretar (Wayne \& Youngs, 2016).

Verificadas las propiedades de un buen instrumento, se utilizó el valor predicho de la primera etapa (Tiempo efectivo de instrucción ${ }_{e, m}$ ) de la ecuación 1 para estimar el modelo de interés entre el desempeño en los componentes de Lenguaje y Matemáticas de la prueba Saber 11 y el tiempo efectivo de instrucción promedio en las escuelas colombianas (ecuación 2), donde el parámetro de interés es $\beta_{1}$ y se espera sea positivo. Lo anterior, debido a que una mayor dedicación a las actividades académicas brinda a los estudiantes más oportunidades de aprender (Bruns \& Luque, 2014). No obstante, es necesario mencionar que una estimación por variables instrumentales provee el efecto local promedio del tratamiento, es decir, se estima el efecto sobre aquellos estudiantes afectados por la variación de los instrumentos.

$$
\text { Saber } \begin{aligned}
11_{\mathrm{i}, \mathrm{e}, \mathrm{m}} & =\beta_{0}+\beta_{1} \text { Tiempo efectivo de instrucción } n_{\mathrm{e}, \mathrm{m}}+\beta_{2} X_{\mathrm{i}, \mathrm{e}, \mathrm{m}} \\
& +\beta_{3} S_{\mathrm{e}, \mathrm{m}}+\gamma_{\mathrm{m}}+U_{\mathrm{i}, \mathrm{e}, \mathrm{m}}
\end{aligned}
$$

En estas ecuaciones, los subíndices $m, e$, e $i$, se refieren a municipio, escuela y estudiante, respectivamente. Por consiguiente, $X_{\mathrm{i}, \mathrm{e}, \mathrm{m}}$ es un conjunto de características del estudiante (i) que asiste a la escuela observada (e) del municipio $(m) S_{\mathrm{e}, \mathrm{m}}$ es un vector de características de cada escuela observada (e) en el municipio $(m)$, que incluye características promedio de los docentes (de Matemáticas o Lenguaje, según el componente a evaluar y de sus estudiantes); 
mientras que $Y_{m}$ son los efectos fijos de municipio $(m)$, incluidos para considerar los efectos socioeconómicos y contextuales que pueden afectar el rendimiento académico de los estudiantes (van der Werf, 2015; García et al., 2014b). Finalmente, $v_{\mathrm{e}, \mathrm{m}} \mathrm{y} v_{\mathrm{i}, \mathrm{e}, \mathrm{m}}$ son los errores estocásticos de ambas ecuaciones.

Un supuesto detrás de esta especificación es que el promedio de las características de los docentes que dictan en grado 11 las asignaturas de interés (Matemáticas y Lenguaje) puede relacionarse con el tiempo promedio de instrucción que se observó en las aulas del mismo grado de cada escuela y este, a su vez, con el desempeño de los estudiantes de estas escuelas en la prueba Saber 11. Como limitante, esta especificación no permite explotar la variabilidad del tiempo de instrucción que se observó al interior de las escuelas y, aún menos, enlazar directamente al docente observado con los resultados de los estudiantes en Saber 11, ya que la información disponible no posibilita tal acción.

\subsubsection{Información utilizada}

Para el desarrollo de esta aproximación se utilizaron los datos de observación en el aula recolectados en el marco del estudio realizado por Bruns y Luque (2014) para el Banco Mundial. Esta base de datos fue recolectada en 2011 y provee información de 2086 aulas de grados 5, 9 y 11, en una muestra de 283 instituciones educativas del país, representativa tanto en el departamento de Antioquia como a nivel nacional. En la presente investigación se emplearon los datos de observaciones de las 673 aulas de grado 11 realizadas en 278 escuelas del país (ver tabla 1). La selección de las aulas a observar fue realizada de forma aleatoria, del mismo modo en que se hizo la selección de las escuelas, mediante un muestreo estratificado (Bruns \& Luque, 2014). Estas no eran informadas de su elección y los profesores solo recibían esta información momentos antes de la observación, junto con la garantía de su anonimato (Bruns \& Luque, 2014); sin embargo, estos autores anotan que los docentes estaban enterados de la presencia del observador $u$ observadores en la clase, lo que podía sesgar su esfuerzo presumiblemente hacia arriba.

De igual forma, se utilizaron los anexos 3A y 5A de la resolución R-166 del Ministerio de Educación Nacional (MEN), que proveen información censal de características sobre 260.011 docentes y 9.269 .848 estudiantes del país, respectivamente. A partir de estas bases de datos se construyen características a nivel de colegio como la proporción de profesores por nivel educativo alcanzado y de estudiantes nuevos, cabezas de familia o repitentes, entre otras. De la misma manera, se emplearon los resultados a nivel de estudiantes de la prueba Saber 11 del año 2011 de las escuelas observadas. Estas bases fueron empalmadas utilizando el código DANE de cada institución educativa (ver tabla 1). 
Tabla 1. Resumen bases de datos utilizadas

\begin{tabular}{|c|c|c|c|c|c|}
\hline Nombre & Agregación & Observaciones & Año & Fuente & $\begin{array}{l}\text { Variable de } \\
\text { empalme }\end{array}$ \\
\hline Saber 11 & Estudiantes & 540.490 & 2011 & ICFES & - \\
\hline $\begin{array}{l}\text { Observacio- } \\
\text { nes de aula }\end{array}$ & Aulas & $\begin{array}{l}\text { - } 2086 \text { aulas en } 283 \text { institucio- } \\
\text { nes } \\
\text { - } 673 \text { aulas de grado } 11 \text { en } \\
278 \text { escuelas, de las cuales } 174 \\
\text { aulas (en } 142 \text { escuelas) fueron } \\
\text { observadas cuando se dictaba } \\
\text { Lenguaje y } 181 \text { cuando se daba } \\
\text { Matemáticas (en } 156 \text { escuelas) }\end{array}$ & 2011 & $\begin{array}{l}\text { Banco } \\
\text { Mundial }\end{array}$ & $\begin{array}{l}\text { Código DANE } \\
\text { de institución } \\
\text { educativa }\end{array}$ \\
\hline $\begin{array}{l}\text { R-166 Anexo } \\
\text { 3A docentes }\end{array}$ & Docentes & 315.917 & 2011 & MEN & $\begin{array}{l}\text { Código DANE } \\
\text { de institución } \\
\text { educativa }\end{array}$ \\
\hline $\begin{array}{l}\text { R-166 Anexo } \\
\text { 5A estudiantes }\end{array}$ & Estudiantes & 9.269 .848 & 2011 & MEN & $\begin{array}{l}\text { Código DANE } \\
\text { de institución } \\
\text { educativa }\end{array}$ \\
\hline
\end{tabular}

Fuente: elaboración propia con datos del Banco Mundial, ICFES, y MEN.

Algunas estadísticas descriptivas de las variables empleadas en esta investigación se encuentran resumidas en los anexos 1 y 2 del presente documento. Estas fueron agrupadas a nivel de estudiantes y sus familias, y de establecimiento educativo. Al final, se contó con información de 11.734 estudiantes de escuelas públicas que presentaron la prueba Saber 11 en 2011, en cuyas instituciones educativas se desarrollaron observaciones de aula, estudiaban de lunes a viernes en jornada matutina, vespertina o completa, y cuya información era consistente. En las estimaciones para el desempeño en Matemáticas, el número de estudiantes se reduce de las mencionadas 11.734 observaciones a 11.630, mientras que en Lenguaje lo hace a 10.725, estos se debió a que las características de los docentes de cada componente evaluado se consolidaron para las escuelas en las que se observó la materia respectiva.

En la figura 1 se detalla cómo se utiliza el tiempo de clases en los grados 11 del país. El panel A muestra que el porcentaje del tiempo dedicado a actividades académicas asciende a un $66 \%$ y que el $12 \%$ del tiempo los profesores se encontraban en tareas ajenas a las relacionadas con el desarrollo de las clases о su manejo. Por su parte, el panel в muestra la cantidad de veces en que el observador registró al docente realizando actividades académicas en cada una de las diez instantáneas del método de observación, esto es el eje $x$, mientras que el eje $y$ expresa el porcentaje de aulas por cantidad de estas observaciones.

Esta es una historia un poco diferente a la del panel A y trata de la variabilidad del tiempo dedicado a estas actividades por los docentes del país. Arriba 
se mencionó que Colombia es la nación latinoamericana, entre las analizadas por Bruns y Luque (2014), en la que más tiempo se dedica a instrucción con un $65 \%$, un punto porcentual por encima de Brasil y Honduras, y alrededor de 3 más que Jamaica (Bruns \& Luque, 2014). Sin embargo, el resultado dista de ser homogéneo dentro de las aulas observadas y en el 5,7\% de las mismas el docente dedicó $100 \%$ del tiempo de clase a actividades académicas (el equivalente a 10 instantáneas). Como contraparte, en el 1,4\% de las aulas el docente destinó un $10 \%$ de la clase a estas actividades.

A. Distribución del uso del tiempo de clase

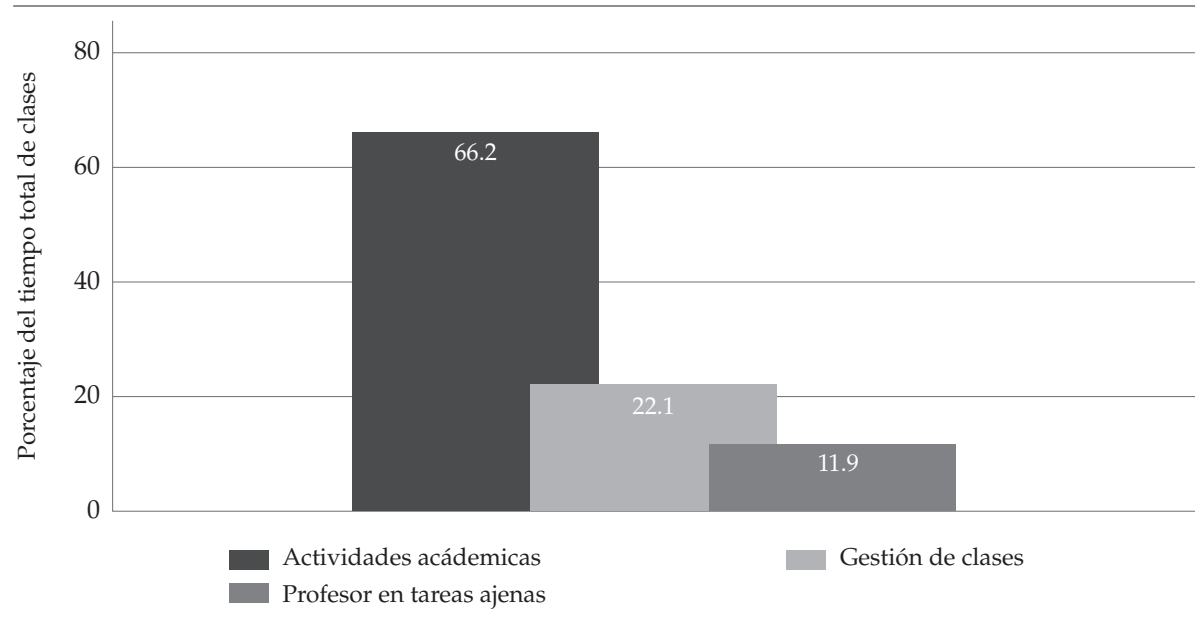

B. Docente en actividades académicas

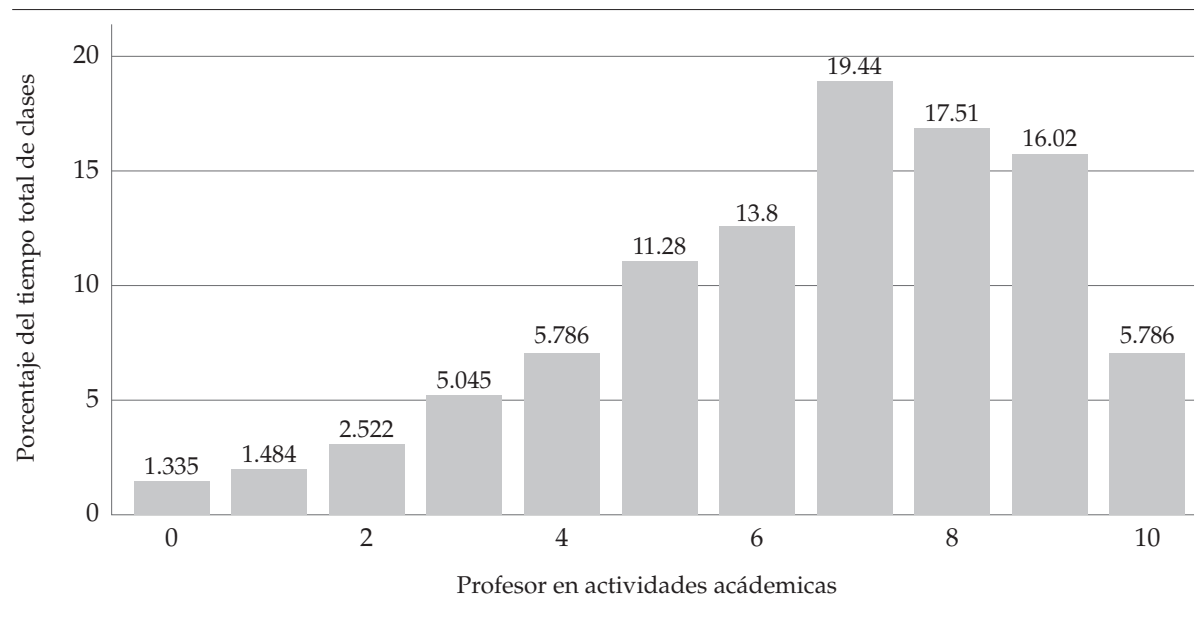

Figura 1. Uso del tiempo de clase en grado 11, Colombia (2011)

Fuente: cálculos del autor con datos del Banco Mundial. 
Llegado a este punto, cabe preguntarse si esta base de datos brinda información sobre la relación entre el porcentaje del tiempo efectivo de instrucción en los grados 11 de las escuelas observadas, en Matemáticas y Lenguaje, y el desempeño académico de sus estudiantes medido por el puntaje promedio en la prueba Saber 11 del año 2011. La figura 2 ilustra la relación entre estas dos variables y la predicción lineal entre las mismas (línea roja). Particularmente, el panel A muestra el desempeño promedio de los estudiantes de esas escuelas en el componente de Matemáticas, mientras que el panel B lo hace para Lenguaje. Para ambos componentes de la prueba se observa una ligera relación positiva; sin embargo, esta representación no dice nada sobre la relación causal entre ambos elementos y pueden estar subestimando (o sobrestimando) la magnitud de la relación.

\section{A. Saber 11 - Matemáticas}

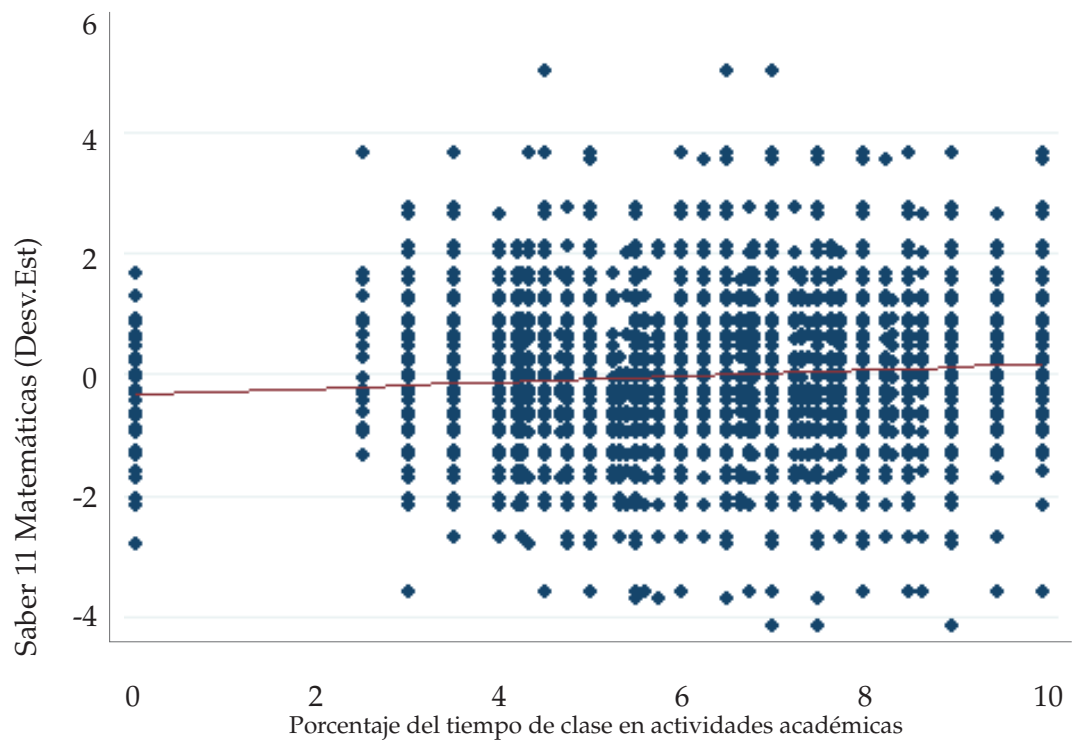




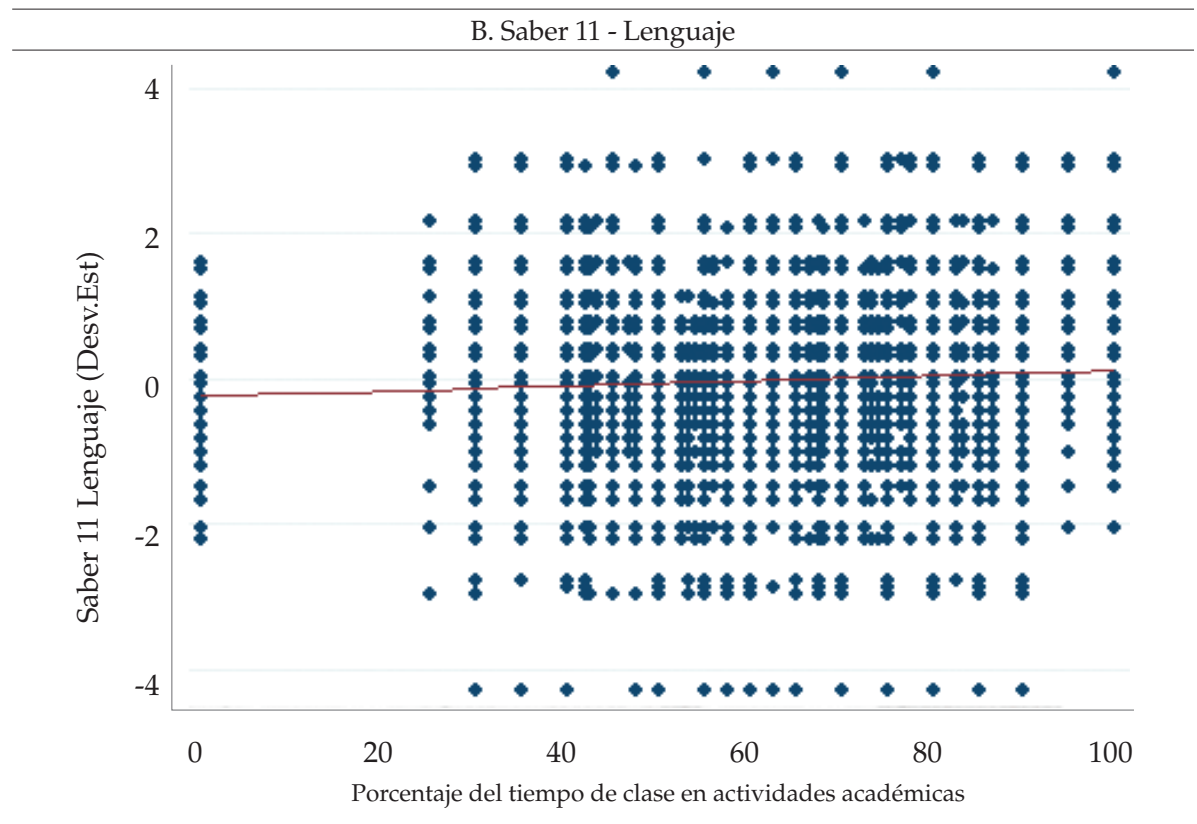

Figura 2. Tiempo efectivo promedio de instrucción en grado 11 y puntaje promedio en la prueba Saber 11, Colombia (2011)

Fuente: cálculos del autor con datos del Banco Mundial y el ICFEs.

\subsection{Aproximación cualitativa}

Con el fin de enriquecer el análisis realizado mediante el uso de herramientas econométricas y la teoría económica se aplicó una aproximación cualitativa; así, el trabajo se alinea con investigaciones en educación realizadas por autores como García et al. (2013) y Palacios et al. (2015), entre otros. Estas metodologías complementan la evidencia cuantitativa, lo que permitió entender mejor cómo "la magia de la educación -la transformación de los insumos educativos en resultados de aprendizaje- sucede en el aula de clases" (Bruns \& Luque, 2014, p. 97)

Por consiguiente, se siguió el enfoque de García et al. (2013) y se aplicaron entrevistas semiestructuradas a docentes que dictan las materias de Matemáticas y Lenguaje a estudiantes de los grados 10 y 11, en las cuales se consultó cómo utilizan el tiempo en el aula, los factores que lo afectan, las consecuencias que perciben acerca del empleo del tiempo y sus propuestas de mejora. ${ }^{9}$

8 Traducción propia.

9 En el Anexo 7 se puede observar el instrumento cualitativo empleado, que incluye el consentimiento informado comunicado a los docentes. 
De esta forma, el diseño de la investigación es de tipo explicativo secuencial, al profundizar y entender mejor los resultados obtenidos mediante la aproximación cuantitativa con los cualitativos (Creswell, 2009; Hernández Sampieri, 2014).

Para implementar este diseño se dividieron los municipios del departamento del Atlántico en dos grupos. El primero estaba compuesto por municipios con más del 50 \% de su población con Necesidades Básicas Insatisfechas (NBI) según el Departamento Administrativo Nacional de Estadística (DANE) y el segundo, con un porcentaje menor al $30 \%$. En cada uno se eligió el municipio con menor cantidad de habitantes que, a su vez, ofreciera la mayor diferencia en el desempeño promedio en la prueba Saber 11 en dos de sus escuelas. Así mismo, se consideró la ubicación de estas dentro del casco urbano del municipio y su cercanía geográfica para reducir la heterogeneidad de la población atendida; los participantes fueron dos profesores de grados 10 y 11 por cada establecimiento elegido -ocho participantes en total-, quienes tenían a su cargo las asignaturas de Lenguaje y Matemáticas. Las transcripciones de las entrevistas fueron codificadas y analizadas con el software NVivo11.

\section{Resultados}

Esta sección consolida y analiza las estimaciones asociadas a la investigación. Los resultados de la primera etapa de mc2e de las estimaciones de interés (resultados en Lenguaje y Matemáticas en Saber 11) se resumen en la tabla 2, ${ }^{10}$ en la cual se observa que las variables instrumentales fueron significativas

10 Características de docentes por establecimiento: proporción de docentes sin título, con título de bachiller pedagógico, con título de normalista, con título de bachiller no pedagógico, con título tecnólogo, con título profesional de licenciado en educación, con título profesional no licenciado en educación, con posgrado en educación, con posgrado pero no en educación; proporción de nombrados en propiedad, provisionales en vacante definitiva, provisionales en vacante provisional, de nombramiento en periodo de prueba; lo anterior, para Matemáticas y Lenguaje, dependiendo de cada regresión. Características de establecimiento: tiempo promedio efectivo de instrucción, edad promedio de los estudiantes, proporción de estudiantes nuevos, proporción de estudiantes cabeza de familia, proporción de estudiantes repitentes, número de estudiantes en grado 11, promedio del colegio en matemáticas en Saber 11 de 2010, varianza del colegio en matemáticas en Saber 11 de 2010, promedio del colegio en Lenguaje en Saber 11 de 2010, varianza del colegio en Lenguaje en Saber 11 de 2010. Características del estudiante y su familia: puntaje Saber 11 Matemáticas, puntaje Saber 11 Lenguaje, sexo, edad, etnia, trabaja, madre sin educación, madre con primaria incompleta, madre con primaria completa, madre con secundaria incompleta, madre con secundaria completa, madre técnica/tecnológica incompleta, madre técnica/tecnológica completa, madre profesional incompleta, madre profesional completa, madre con posgrado, estrato 1 , estrato 2 , estrato 3 , estrato 4 , estrato 5 , estrato 6 , asiste en jornada completa, asiste en jornada mañana, asiste en jornada tarde. 
al nivel del 1\%, lo que indica su relevancia individual (Rosales et al., 2013; Wooldridge, 2002). La relevancia conjunta fue evaluada con la prueba F de los instrumentos (Bernal \& Peña, 2011), la cual tuvo un valor de 68,34 en la estimación de Matemáticas y de 67,10 en la de Lenguaje, valores estadísticamente significativos al nivel del 1\%. Así mismo, los valores de 94,39 en la especificación para Matemáticas y 156,56 en la de Lenguaje de la prueba Cragg-Donald permitieron descartar la hipótesis de instrumentos débiles. Previamente, estos instrumentos fueron expuestos a la prueba de sobreidentificación de Sargan, la cual mostró ambos como válidos en las especificaciones de los dos componentes analizados (p-valor de 0,21 y 0,53 en Matemáticas y Lenguaje, respectivamente). Se aplicó el test de endogeneidad de Hausman y se verificó dicho problema para la variable de interés en el modelo de Matemáticas, pero no para la de Lenguaje. ${ }^{11}$

Tabla 2. Resultados de estimación de las primeras etapas, tiempo efectivo de instrucción e instrumentos -edad promedio de docentes y su polinomio al cuadrado-

\begin{tabular}{lcc}
\hline & Matemáticas & Lenguaje \\
\hline Edad promedio de los docentes & $-1.747^{* * *}$ & $-4.063^{* * *}$ \\
& $(0.234)$ & $(0.351)$ \\
Edad promedio de los docentes al cuadrado & $1.624^{* * *}$ & $4.157^{* * *}$ \\
Características de docentes por establecimiento & $(0.237)$ & $(0.359)$ \\
Efectos fijos de municipio & Sí & Sí \\
Características de establecimiento & Sí & Sí \\
Características del estudiante y su familia & Sí & Sí \\
Observaciones & Sí & 10,725 \\
R-cuadrado & 11,630 & 0.721 \\
\hline
\end{tabular}

Errores estándar robustos en paréntesis ${ }^{* * *} \mathrm{p}<0.01,{ }^{* *} \mathrm{p}<0.05,{ }^{*} \mathrm{p}<0.1$

Fuente: cálculos del autor con datos del Banco Mundial, ICFES, y MEN.

A su vez, la tabla 3 resume los resultados de las estimaciones entre el tiempo efectivo de instrucción y el desempeño de los estudiantes en el componente de Matemáticas para el 2011. Allí se consideraron diferentes especificaciones

11 Los resultados corresponden a la estimación en Stata empleando el comando ivreg2. Ver Anexo 3 para un resumen del test de Hausman y Anexo 4 para los resultados de una estimación de la prueba de sobreidentificación que sigue las indicaciones de Rosales et al. (2013). 
dadas por los diversos grupos de controles analizados, ${ }^{12}$ tanto para мсо como para MC2E. No obstante, el análisis se centrará en la cuarta y octava columna, dado que solo sobre las estimaciones de MC2E puede inferirse causalidad entre las variables, debido al problema de endogeneidad tratado en la sección metodológica de este trabajo. De esta forma, se observa en la columna cuatro que aumentar en una desviación estándar el porcentaje de tiempo efectivo de instrucción en las escuelas analizadas está asociado con un incremento de 0,04 desviaciones estándar en el puntaje promedio de los estudiantes en Matemáticas. Estos resultados son estadísticamente significativos al nivel del 1\%.

Tabla 3. Efecto del tiempo efectivo de instrucción en los resultados de Matemáticas en la prueba Saber 11, Colombia (2011) ${ }^{13}$

\begin{tabular}{|c|c|c|c|c|c|c|c|c|}
\hline & \multicolumn{4}{|c|}{ мСо } & \multicolumn{4}{|c|}{ MC2E } \\
\hline & (1) & (2) & (3) & $(4)$ & (5) & (6) & (7) & (8) \\
\hline \multirow{2}{*}{$\begin{array}{l}\text { Tiempo efectivo de ins- } \\
\text { trucción }\end{array}$} & $0.075^{* * *}$ & $0.091^{* * *}$ & $0.066^{* * *}$ & $0.047^{* * *}$ & $0.459^{* * *}$ & $1.075^{* * *}$ & $0.474^{* * *}$ & $0.431^{* * *}$ \\
\hline & $(0.010)$ & $(0.014)$ & $(0.015)$ & $(0.015)$ & $(0.068)$ & $(0.367)$ & $(0.141)$ & $(0.118)$ \\
\hline $\begin{array}{l}\text { Características de docen- } \\
\text { tes por establecimiento }\end{array}$ & Sí & Sí & Sí & Sí & Sí & Sí & Sí & Sí \\
\hline Efectos fijos de municipio & & Sí & Sí & Sí & & Sí & Sí & Sí \\
\hline $\begin{array}{l}\text { Características de esta- } \\
\text { blecimiento }\end{array}$ & & & $\mathrm{Si}$ & $\mathrm{Si}$ & & & Sí & Sí \\
\hline $\begin{array}{l}\text { Características del estu- } \\
\text { diante y su familia }\end{array}$ & & & & Sí & & & & Sí \\
\hline Observaciones & 11,630 & 11,630 & 11,630 & 11,630 & 11,630 & 11,630 & 11,630 & 11,630 \\
\hline R-cuadrado & 0.037 & 0.139 & 0.156 & 0.199 & & & 0.101 & 0.152 \\
\hline
\end{tabular}

Errores estándar robustos en paréntesis ${ }^{* * *} \mathrm{p}<0.01,{ }^{* *} \mathrm{p}<0.05,{ }^{*} \mathrm{p}<0.1$

Fuente: cálculos del autor con datos del Banco Mundial, ICFEs, y MEN.

Sin embargo, se argumentó que una estimación por mco produciría resultados sesgados, por lo que se emplearon MC2E para resolver dicho problema. En consecuencia, en la columna ocho se puede apreciar que el efecto de aumentar el tiempo efectivo de instrucción promedio en una desviación estándar incrementa en 0,43 desviaciones estándar los puntajes promedios de los estudiantes en Matemáticas. Esto equivale a un aumento de 2,67 puntos

12 Las variables continúas fueron estandarizadas con media cero y desviación estándar igual a uno.

$13 \mathrm{El}$ anexo 5 ofrece la versión completa de estos resultados. Descripción de controles en nota al pie número 9 . 
en este componente de la prueba Saber 11, por un aumento del $10 \%$ en el tiempo efectivo de instrucción que reciben los estudiantes en sus clases. Un efecto 10 veces mayor al estimado por mco, estadísticamente diferente de cero al nivel del 1\%, y que sí puede ser interpretado causalmente. En este punto puede pensarse que el canal de transmisión entre el tiempo efectivo de instrucción y el desempeño académico de los estudiantes posibilita las mayores oportunidades de aprendizaje en el aula.

Ahora bien, los resultados para el componente de Lenguaje son menores a los observados en Matemáticas. En la tabla 4 se advierte, según la estimación por MC2E correspondiente a la columna ocho, que aumentar en una desviación estándar el tiempo efectivo de instrucción promedio ocasiona un aumento de 0,16 desviaciones estándar en el desempeño de los estudiantes en Lenguaje. A un nivel de confianza del $10 \%$, este coeficiente es diferente a cero y triplica al obtenido mediante la especificación no causal de la columna cuatro por MCO (0,05 desviaciones estándar). Este puede expresarse como una ganancia de 0,95 puntos en esta área de la prueba Saber 11 por cada 10 puntos porcentuales de aumento en el tiempo efectivo de instrucción promedio. La intuición detrás de la diferencia en la importancia del tiempo efectivo de instrucción

Tabla 4. Efecto del tiempo efectivo de instrucción en los resultados de Lenguaje en la prueba Saber 11, Colombia (2011) ${ }^{14}$

\begin{tabular}{|c|c|c|c|c|c|c|c|c|}
\hline & \multicolumn{4}{|c|}{ Mсо } & \multicolumn{4}{|c|}{ MC2E } \\
\hline & (1) & (2) & (3) & $(4)$ & (5) & (6) & (7) & (8) \\
\hline \multirow{2}{*}{$\begin{array}{l}\text { Tiempo efectivo de ins- } \\
\text { trucción }\end{array}$} & $0.059^{* * *}$ & $0.094^{* * *}$ & $0.051^{* * *}$ & $0.050^{* * *}$ & $0.765^{* * *}$ & $1.040^{* * *}$ & $0.194^{* *}$ & $0.158^{*}$ \\
\hline & $(0.011)$ & $(0.016)$ & $(0.017)$ & $(0.017)$ & $(0.116)$ & $(0.168)$ & $(0.094)$ & $(0.094)$ \\
\hline $\begin{array}{l}\text { Características de docen- } \\
\text { tes por establecimiento }\end{array}$ & Sí & Sí & Sí & Sí & Sí & Sí & Sí & Sí \\
\hline Efectos fijos de municipio & & Sí & Sí & Sí & & Sí & Sí & Sí \\
\hline $\begin{array}{l}\text { Características de esta- } \\
\text { blecimiento }\end{array}$ & & & Sí & Sí & & & Sí & Sí \\
\hline $\begin{array}{l}\text { Características del estu- } \\
\text { diante y su familia }\end{array}$ & & & & Sí & & & & Sí \\
\hline Observaciones & 10,725 & 10,725 & 10,725 & 10,725 & 10,725 & 10,725 & 10,725 & 10,725 \\
\hline R-cuadrado & 0.044 & 0.139 & 0.154 & 0.182 & & & 0.149 & 0.179 \\
\hline
\end{tabular}

Errores estándar robustos en paréntesis ${ }^{* * *} \mathrm{p}<0.01,{ }^{* *} \mathrm{p}<0.05,{ }^{*} \mathrm{p}<0.1$

Fuente: cálculos del autor con datos del Banco Mundial, ICFEs, y MeN.

14 El Anexo 6 ofrece la versión completa de estos resultados. Descripción de controles en nota al pie número 9. 
en los puntajes de Matemáticas y Lenguaje es que, en el primer caso, puede ser más importante el desarrollo de explicaciones, ejercicios en clase y actividades de monitoreo al progreso de los estudiantes en las temáticas, mientras que en el segundo caso los hábitos de lectura aprendidos en el hogar pueden jugar un papel más importante que las clases en sí, más aún si se considera que el coeficiente para Lenguaje es apenas estadísticamente significativo y que pierde parte de esta al controlar por las características del hogar.

Los resultados obtenidos son un aporte a la literatura económica internacional sobre educación del lado de la oferta, en la cual la evidencia es más débil que en el de la demanda (Banerjee, Glewwe, Powers \& Wasserman, 2013). Estos estudios sugieren que si el sistema educativo colombiano alcanza el porcentaje del tiempo efectivo de instrucción del marco de referencia estadounidense, es decir, logra pasar de $65 \%$ a $85 \%$ según los cálculos de Bruns \& Luque (2014), sus estudiantes podrían obtener ganancias promedio de 5,34 puntos en los resultados de la prueba Saber 11 en Matemáticas y de 1,9 en Lenguaje.

Para ilustrar, los resultados en el componente de matemáticas son comparables con el incremento en 0,4 desviaciones estándar en la prueba Saber 11 que se obtendría al disminuir el nivel de intimidación escolar en Colombia en una desviación estándar, según las estimaciones de van der Werf (2014). Aunque es necesario recordar que esta investigación se centró en el uso eficiente del tiempo de clase disponible y no en la cantidad de horas de clase semanales, los resultados aquí obtenidos superan el aumento en los puntajes de estudiantes de grado noveno que asistían a colegios de jornada completa estimado por Hincapié (2016) de 0,110 y 0,138 desviaciones estándar en Lenguaje y Matemáticas, respectivamente. De forma análoga, son mayores que el efecto de 0,2 desviaciones estándar logrado cuando los estudiantes son beneficiarios del Programa de Ampliación de Cobertura de la Educación Secundaria (PACES), como lo mostraron los resultados promedio de una prueba aplicada a una submuestra por Angrist, Bettinger, Bloom, King y Kremer (2002).

A nivel internacional, Bellei (2009) estima el efecto de la implementación de la jornada única en Chile entre 0,05-0,07 y 0,00-0,12 desviaciones estándar en Lenguaje y Matemáticas, respectivamente. Mientras que Lavy (2012) obtuvo que aumentar en una hora semanal las clases de Matemáticas, Ciencias e Inglés aumenta en promedio los resultados en pruebas en 0,053 desviaciones estándar. Así mismo, intervenciones para reducir el ausentismo docente en India y Kenia han mostrado efectos de 0,21 desviaciones estándar en lenguaje (Duflo et al., 2012) y de 0,16-0,21 desviaciones estándar en matemáticas (Duflo et al., 2015, 2012). 
Ahora bien, hay que reiterar que los docentes estaban enterados de la presencia del observador en el aula, lo cual pudo hacer que se esforzaran más, afectando la cantidad de tiempo efectivo de instrucción que impartieron (Bruns \& Luque, 2014). Esta situación puede impedir que el sesgo en las estimaciones desaparezca totalmente. Por otra parte, las tablas 3 y 4 muestran que los coeficientes estimados mediante MC2E son sistemáticamente superiores a los de MCO, comportamiento consistente con los resultados de Bonilla (2011) y van der Werf (2014). Lo anterior puede deberse a que el efecto de la variable de interés o el tratamiento puede ser mayor entre aquellos estudiantes con una tasa marginal de retorno más alta que el promedio (Harmon, Oosterbeek \& Walker, 2000), es decir, si se diera el caso de que un incremento del tiempo efectivo de instrucción beneficiara más a aquellos estudiantes con un rendimiento académico menor.

\subsection{Resultados cualitativos}

La aplicación de las entrevistas a docentes permitió identificar algunos factores asociados al uso del tiempo efectivo de instrucción en las escuelas objeto del estudio de caso en el departamento del Atlántico. Sin embargo, es necesario recordar que estos resultados solo arrojan una luz detrás de la relación entre estas variables, pero su aplicabilidad a otras poblaciones debe ser analizada con precaución. Concretamente, los docentes entrevistados son conscientes de la importancia que tiene usar de forma eficiente el tiempo del que disponen; parafraseando sus palabras, para desarrollar los contenidos completamente, alcanzar los objetivos demandados por el Gobierno y no perder la continuidad en el desarrollo de las temáticas para evitar nuevas pérdidas de tiempo. Con respecto a este último elemento, uno de los docentes afirmó que: “es posible que el tiempo marque en qué momentos un estudiante desarrolla algunos procesos de aprendizajes y de qué manera ese proceso, que lleva un orden, se interrumpa y que retomarlo quizá no es fácil" (Entrevista a docente de colegio de bajo desempeño y bajo NBI, 5 de septiembre de 2016). Considerando que, en su mayoría, los docentes manifestaron que el tiempo del que disponen es insuficiente para cubrir los contenidos, aumentar el tiempo efectivo de instrucción contribuiría a mejorar esta situación.

No obstante, los docentes identificaron variados elementos que afectan el desarrollo normal de sus clases y les impiden dedicar un mayor porcentaje del tiempo de las mismas a actividades académicas. En este sentido, la disciplina parece ser el principal obstáculo y apareció de manera repetida en casi la totalidad de las entrevistas realizadas. Particularmente, en la institución 
de bajo desempeño del municipio de alto NBI se mencionó la proclividad de los estudiantes a empezar riñas, algo que no parece ser particularmente importante en la institución de mejores resultados del mismo municipio. Así mismo, fue indicado por un docente en la institución de alto desempeño y bajo NBI que: "[...] hay que interrumpir la clase porque los que están en la clase no escuchan lo que estamos trabajando, porque hay demasiado ruido" (Entrevista a docente de colegio de alto desempeño y bajo NBI, 18 de octubre de 2016).

Otro elemento recurrente en las escuelas de bajo rendimiento académico de ambos municipios son las condiciones de ventilación de las aulas y la temperatura en la que se desarrollan las clases: "[...] el calor, no hay abanico, se va la luz y, obviamente, uno entra a un salón donde la temperatura está casi a 30 y pico de grados, entonces es muy pesado" (Entrevista a docente de colegio de bajo desempeño y alto NBI, 5 de septiembre de 2016). De la misma manera, en el municipio de bajo NBI un docente manifestó: "cuando hay mucho calor, los estudiantes no soportan estar 42 o 45 personas metidas en un solo salón con los abanicos en malas condiciones" (Entrevista a docente de colegio de bajo desempeño y bajo NBI, 18 de octubre de 2016); lo anterior genera interrupciones a las actividades y reducción del tiempo de clase para evitar la fatiga y/o sofocación de estudiantes y docentes.

En contraste, en las escuelas de puntajes más altos esos elementos no fueron mencionados y los profesores se inclinaron por la insuficiencia de elementos de apoyo, tales como proyectores y dispositivos electrónicos para los estudiantes, que les permitan usar otras estrategias de enseñanza. Con respecto a lo anterior, la logística necesaria para su instalación o la distribución de su uso entre los diferentes miembros de la comunidad académica consume el tiempo de las clases: "[...] no todas las aulas tienen, digamos, tableros inteligentes, entonces tú te tienes que desplazar de un lugar a otro y permitir o cambiar con un docente para presentarles a ellos [los estudiantes] algo en un videobeam o utilizar las tablets. En este caso, te tienes que dirigir a la sala de bilingüismo" (Entrevista a docente de colegio de alto desempeño y bajo NBI, 18 de octubre de 2016).

Los docentes de ambas instituciones del municipio de alto NBI también mencionaron que los desplazamientos hacia la escuela de estudiantes y profesores que residen fuera del municipio afectan el desarrollo normal de las primeras horas de clase, debido a retrasos e inconvenientes, como bloqueos de las vías. Por el contrario, este elemento no fue mencionado en el municipio de bajo NBI. Otro elemento mencionado son las interrupciones generadas por la realización de actividades extracurriculares, tales como reuniones no 
programadas, charlas financieras de agentes bancarios, informaciones de estudiantes, llamados de las directivas y eventos de la Secretaría de Educación o el Ministerio de Educación Nacional.

Finalmente, la atención a los padres de familia aparece como un elemento que interrumpe las clases en las instituciones de bajo desempeño de ambos municipios, donde "[...] el padre de familia llega directamente al salón a buscarte" (Entrevista a docente de colegio de bajo desempeño y alto NBI, 5 de septiembre de 2016). Esto no parece suceder en los colegios de mejor desempeño del municipio de alto NBI debido a que "[...] los padres de familia cuando, de pronto, quieren preguntarle algo a los profesores aprovechan el espacio del receso [...]" (Entrevista a docente de colegio de alto desempeño y alto NBI, 5 de septiembre de 2016). Tampoco parece ser problema en la institución de alto desempeño del municipio de bajo NBI, donde no fue mencionado.

\section{Conclusiones y recomendaciones de política pública}

La relación entre el tiempo efectivo de instrucción y el desempeño académico de los estudiantes es un área de investigación con territorio por explorar. Esto, dentro de una coyuntura de reformas educativas en el país, marcadas por pobres resultados en pruebas internacionales y restricciones fiscales, demanda más aproximaciones para que quienes toman las decisiones elijan con la mayor información posible. Por consiguiente, empleando la información sobre observaciones de aula recolectada por el Banco Mundial y mediante una aproximación de variables instrumentales se estimó causalmente la relación mencionada.

Los resultados sugieren que un incremento de una desviación estándar en el tiempo efectivo de instrucción promedio aumenta en 0,43 desviaciones el puntaje promedio en Matemáticas de la prueba Saber 11, resultado estadísticamente diferente de cero al nivel del $1 \%$, mientras que en el componente de Lenguaje el aumento es de 0,16 desviaciones con un nivel de confianza del $10 \%$. Lo anterior se traduce en que un aumento del $10 \%$ en el tiempo efectivo de instrucción promedio que reciben los estudiantes aumenta en 2,67 y 0,95 puntos los resultados en los componentes de Matemáticas y Lenguaje de la prueba Saber 11, respectivamente. En consecuencia, puede concluirse que el tiempo efectivo de instrucción es relativamente más importante para el desarrollo de las habilidades evaluadas en Matemáticas de los estudiantes colombianos que las de Lenguaje, donde quizá primen los hábitos de lectura aprendidos en el hogar. 
De forma comparativa, los resultados obtenidos en el componente de Matemáticas son similares en magnitud al efecto que tendría disminuir el nivel de intimidación escolar en Colombia en una desviación estándar (van der Werf, 2014), mientras que son superiores al efecto de la jornada completa en el país para noveno grado (Hincapié, 2016) y el del PACEs (Angrist et al., 2002). Así mismo, las ganancias aquí estimadas son mayores a las calculadas por Bellei (2009) para la implementación de la jornada única en Chile en Lenguaje y Matemáticas, respectivamente, y las de Lavy (2012) para extender en una hora semanal las clases de Matemáticas, Ciencias e Inglés. Por otra parte, intervenciones para reducir el ausentismo docente en India y Kenia han mostrado efectos de 0,21 desviaciones estándar en Lenguaje (Duflo et al., 2012) y de 0,16-0,21 desviaciones estándar en matemáticas (Duflo et al., 2015 , 2012). En consecuencia, se subraya la atención que debe recibir el tiempo efectivo de instrucción como variable relevante en el mejoramiento de los resultados académicos de los estudiantes colombianos; es necesario generar más estudios acerca de esta relación, los mecanismos para incrementar el tiempo de instrucción y su contraste con otras opciones de política pública educativa.

Por otra parte, los resultados de la aproximación cualitativa realizada mediante el caso de estudio en el departamento del Atlántico sugieren que la disciplina es un elemento que afecta el tiempo efectivo de instrucción en todas las escuelas visitadas. Por su parte, la ventilación y la temperatura fueron manifestados repetidamente como un obstáculo en el desarrollo de las clases en las escuelas de bajo rendimiento, algo que no parece ocurrir en las de mejores puntajes. Análogamente, aspectos como los desplazamientos desde lugares diferentes al municipio de la escuela, la realización de actividades ajenas a las clases y las interrupciones de los padres, aparecen como elementos a ser considerados a la hora de buscar mejoras en el tiempo efectivo de instrucción, pero pueden ser intervenidos desde la comunidad educativa propia de cada institución. Sin embargo, las condiciones de las aulas y de desplazamiento hacia la institución tanto de docentes como de estudiantes requieren de una coordinación con entidades de nivel superior para el acopio de los recursos necesarios y la generación de acciones correctivas. Los docentes reconocen que el tiempo del que disponen es insuficiente para cubrir adecuadamente los contenidos de las asignaturas, razón por la que reducir la afectación generada por estos factores puede contribuir a aumentar el tiempo efectivo de instrucción en las escuelas y el desempeño de los estudiantes; no obstante, se subraya nuevamente que los resultados de esta sección deben ser analizados con precaución al intentar generalizarlos a otras poblaciones. 
Finalmente, respecto a las implicaciones de política, los resultados de este estudio se ofrecen como una oportunidad para analizar las actuales políticas educativas del país y encontrar en el tiempo efectivo de instrucción una alternativa de solución a sus deficiencias educativas. Por lo tanto, es necesario estudiar a profundidad las actividades que, consideradas como tiempo efectivo de instrucción, pueden mejorar el logro académico de los estudiantes. ${ }^{15}$ Así mismo, es importante desarrrollar un análisis de costo-efectividad de la implementación de posibles acciones que busquen mejoras en el tiempo efectivo de instrucción, con el fin de contrastar con otras alternativas, como la jornada única. En este sentido, la ventilación o climatización de las aulas, las mejoras disciplinarias, el establecimiento de horarios de atención a padres, la minimización de distancias y / o tiempos de traslado residencia-escuela de los docentes y estudiantes, son elementos que pueden brindar soluciones. Este trabajo es una mirada inicial y deben considerarse futuras investigaciones sobre los determinantes de esta variable y los canales específicos por los cuales afecta el rendimiento de los estudiantes.

\section{Agradecimientos}

El autor agradece al departamento del Atlántico y a Colciencias por permitirle financiar sus estudios de maestría con la Beca-Crédito para la Formación de Capital Humano para el Departamento del Atlántico de 2014. Así mismo, agradece de manera muy especial a Sandra García y Catherine Rodríguez por su invaluable guía en la elaboración de este documento, presentado inicialmente como requisito de grado del doble programa de la Maestría en Economía y la Maestría en Políticas Públicas de la Universidad de los Andes.

\section{Descargos de responsabilidades}

Todos los errores $\mathrm{u}$ omisiones son responsabilidad del autor.

\section{Referencias bibliográficas}

Abadzi, H. (2009). Instructional time loss in developing countries: Concepts, measurement, and implications. World Bank Research Observer, 24, 267-290. Angrist, J., Bettinger, E., Bloom, E., King, E., \& Kremer, M. (2002). Vouchers for private schooling in Colombia: Evidence from a randomized natural experiment. American Economic Review, 92(5), 1535-1558.

15 Kane et al. (2011) realizan un valioso aporte en esta dirección. 
Banerjee, A., \& Duflo, E. (2006). Addressing absence. Journal of Economic Perspectives, 20(1), 117-132. Doi: https:/ / doi.org/10.1257/089533006776526139

Banerjee, A., Glewwe, P., Powers, S., \& Wasserman, M. (2013). Expanding access and increasing student learning in post-primary education in developing countries: A review of the evidence. Post-Primary Education Initiative Review Paper. Abdul Latif Jameel Poverty Action Lab (J-PAL).

Barrera, F., Maldonado, D., \& Rodríguez, C. (2012). Calidad de la educación básica y media en Colombia: diagnóstico y propuestas. Documentos CEDE.

Bellei, C. (2009). Does lengthening the school day increase students' academic achievement? Results from a natural experiment in Chile. Economics of Education Review, (28), 629-640.

Bernal, R., \& Peña, X. (2011). Guía práctica para la evaluación de impacto (1 edición,). Bogotá, Colombia: Universidad de los Andes, Facultad de Economía, Centro de Estudios sobre Desarrollo Económico.

Bonilla, L. (2011). Doble jornada escolar y calidad de la educación en Colombia. Documentos de Trabajo sobre Economía Regional (143).

Bruns, B., \& Luque, J. (2014). Inside the classroom in Latin America and the Caribbean. En B. Bruns, \& J. Luque, Great teachers. How to raise student learning in Latin American and the Caribbean (advance edition) (pp. 97-138). Washington, Dc: World Bank.

Burgos, J. (2011). Brecha educativa en la población rural y urbana en Colombia (Tesis de pregrado). Universidad del Valle, Santiago De Cali, Colombia.

Cameron, A., \& Trivedi, P. (2005). Linear models. En Microeconometrics methods and aplications (pp. 65-115). Cambridge: Cambridge University Press.

Creswell, J. W. (2009). Research design: Qualitative, quantitative, and mixed methods approaches (3 edición). Thousand Oaks, California: Sage.

DNP (2015). Plan Nacional de Desarrollo 2014-2018. Todos por un Nuevo País. Tomo 1. Bogotá.

Duflo, E., Dupas, P., \& Kremer, M. (2015). School governance, teacher incentives, and pupil-teacher ratios: Experimental evidence from kenyan primary schools. Journal of Public Economics, 123, 92-110. Doi: https:/ / doi.org/10.1016/j.jpubeco.2014.11.008

Duflo, E., Hanna, R., \& Ryan, S. P. (2012). Incentives work: Getting teachers to come to school. The American Economic Review, 102(4), 1241-1278.

García , S., Fernández , C., \& Weiss, C. (2013). Does lengthening the school day reduce the likelyhood of early school dropout and grade repetition: evidence from Colombia. Documentos de Trabajo Escuela de Gobierno Alberto Lleras Camargo (7). 
García, S., Maldonado, D., Perry, G., Rodríguez, C., \& Saavedra, J. (2014a). La contribución de diferentes insumos escolares al logro estudiantil. En Tras la Excelencia Docente (pp. 48-67). Bogotá: Fundación Compartir. García, S., Maldonado, D., Perry, G., Rodríguez, C., \& Saavedra, J. (2014b). La calidad docente y el aprendizaje estudiantil en Colombia. En Tras la Excelencia Docente (pp. 68-79). Bogotá: Fundación Compartir.

García, S., Maldonado, D., Perry, G., Rodríguez, C., \& Saavedra, J. (2014c). Formación, Características y Remuneración. En Tras la Excelencia Docente (pp. 132-175). Bogotá: Fundación Compartir.

García, S., Maldonado, D., Perry, G., Rodríguez, C., \& Saavedra, J. E. (2014d). Los docentes de los colegios oficiales de alto y bajo desempeño. En Tras la Excelencia Docente (pp. 176-195). Fundación Compartir.

Glewwe, P., Hanushek, E., Humpage, S., \& Ravina, R. (2013). School resources and educational outcomes in developing countries: A review of the literature from 1990 to 2010. En P. Glewwe, Education policy in developing countries (pp. 13-64). Chicago and London: The University of Chicago Press.

Goodman, J. (2014). Flaking out: Student absences and snow days as disruptions of instructional time. NBER Working Paper.

Harmon, C., Oosterbeek, H., \& Walker, I. (2000). The returns to education a review of evidence, issues and deficiencies in the literature. Londres: Centre for the Economics of Education.

Hanushek, E. (1971). Teacher characteristics and gains in student achievement: Estimation using micro data. The American Economic Review, 61(2), 280-288. Hanushek, E. (2011). The economic value of higher teacher quality. Economics of Education Review, 30(3) 466-479.

Hanushek, E. (2016). What matters for student achievement. Education Next, 23-30.

Hernández Sampieri, R. (2014). Metodología de la investigación (6 edición). México, D.F.: McGraw-Hill.

Hincapié, D. (2016). Do longer school days improve student achievement? Evidence from Colombia. IDB Working Paper Series.

ICFES (2013). Colombia en PISA 2012. Informe Nacional de Resultados. Resumen Ejecutivo. Bogotá.

Kairuz , V., Correa, C., Durán, L., Godoy, J., \& Perilla, M. (2008). Brechas Educativas de la Población. Censo 2005. Revista de la Información Básica. Kane, T., Taylor, E., Tayler, J., \& Wooten, A. (2011). Identifying effective classroom practices using student achievement data. The Journal of Human Resources, 46(3), 587-613. 
Lavy, V. (2010). Do differences in schools' instruction time explain international achievement gaps? Evidence from developed and depeloping countries. NBER Working Paper.

Lavy, V. (2012). Expanding school resources and increasing time on task: Effects of a policy experiment in israel on student academic achievement and behavior. NBER Working Paper.

Mckee, G., Rivkin, S., \& Sims, K. (2010). Disruption, achievement and the heterogeneous benefits of smaller classes. NBER Working Paper.

Meghir, C., \& Rivkin, S. (2011). Econometric methods for research in education. En E. Hanushek, S. Machin, \& L. Woessmann (Edits.), Handbook of the Economics of Education Vol. 3 (pp. 1-87). Estados Unidos: Elsevier B. v.

Méndez, I. (2013). Prácticas Docentes y Rendimiento Estudiantil: Evidencia a partir de TALIS 2016 y PISA 2012. La Rioja: Consejería de Educación, Cultura y Turismo del Gobierno de la Rioja; Fundación Santillana, Instituto Nacional de Evaluación Educativa.

Mina, A. (2004). Factores asociados al logro educativo a nivel municipal. Documento CEDE.

Muralidharan, K., Das, J., Holla, A., \& Mohpal, A. (2017). The fiscal cost of weak governance: Evidence from teacher absence in India. Journal of Public Economics, 145, 116-135. Doi: https://doi.org/10.1016/j.jpubeco.2016.11.005

Palacios, G., Sánchez, F., \& Córdoba, C. (2015). Etnoeducación y desempeño escolar en la Región Pacífica colombiana. Documentos CEDE (36).

Pokroprek, A. (2016). Introduction to instrumental variables and their application to largescale assessment data. SpringerOpen Journal, 4(1), 1-20.

Resolución 0207 de 2010 (23 de febrero). Por por la cual se reglamentan las audiencias públicas para selección de plaza en institución educativa oficial de conformidad con las listas de elegibles para proveer empleos que se rigen por el sistema especial de Carrera Docente.

Rivkin, S., \& Schiman, J. (2013). Instruction time, classroom quality, and academmic achievement. NBER Working Paper.

Rivkin, S., Hanushek, E., \& Kain, J. (2005). Teachers, schools, and academic achievement. Econometrica, 73(2), 417-458.

Rosales, R., Perdomo, J., Morales, C., \& Urrego, J. (2013). Fundamentos de Econometría Intermedia: Teoría y Aplicaciones. Bogotá: Universidad de los Andes. unesco (2015). Informe de Resultados TERCE. Factores Asociados. Santiago de Chile: unesco.

van der Werf, C. (2014). The effects of bullying on academic achievement. Revista Desarrollo y Sociedad, (74), 275-308. 
Venäläinen, R. (2008). What do we know about instructional time use in Mali? Assessing the suitability of the classroom observation snapshot instrument for use in developing countries. HDNED, World Bank.

Wayne, A., \& Youngs, P. (2016). Teacher characteristics and student achievement gains: A review. Review of Educational Research, 73(1), 89-122.

Woessmann, L. (2016). The importance of school systems: Evidence from international differences in student achievement. Warwick Working Paper Series.

Wooldridge, J. (2002). Instrumental variables estimation of single-equation linear models. En Econometric analysis of cross section and panel data (pp. 83-114). Cambridge: The Miт Press.

World Bank Group (2015). User guide. Conducting clasroom observations.

\section{Anexo 1. Estadísticas descriptivas a nivel de estudiante e institución educativa}

\begin{tabular}{|c|c|c|c|c|c|}
\hline Variable & Observ. & Media & Desv. Est. & Mínimo & Máximo \\
\hline \multicolumn{6}{|l|}{ Estudiante y familia } \\
\hline Puntaje Saber 11 Matemáticas & 11,734 & 46,04 & 10,93 & 0,00 & 101,00 \\
\hline Puntaje Saber 11 Lenguaje & 11,734 & 46,10 & 10,62 & 0,00 & 91,00 \\
\hline Sexo (1=Hombre) & 11,734 & 0,45 & 0,50 & 0,00 & 1,00 \\
\hline Edad & 11,734 & 16,67 & 2,02 & 9,00 & 81,00 \\
\hline Etnia (1=Pertenece a grupo étnico) & 11,734 & 0,04 & 0,20 & 0,00 & 1,00 \\
\hline Trabaja (1=Sí) & 11,734 & 0,07 & 0,26 & 0,00 & 1,00 \\
\hline \multicolumn{6}{|l|}{ Educación de la madre (1=Sí) } \\
\hline Madre sin educación & 11,734 & 0,02 & 0,13 & 0,00 & 1,00 \\
\hline Madre con primaria incompleta & 11,734 & 0,19 & 0,39 & 0,00 & 1,00 \\
\hline Madre con primaria completa & 11,734 & 0,20 & 0,40 & 0,00 & 1,00 \\
\hline Madre con secundaria incompleta & 11,734 & 0,20 & 0.40 & 0,00 & 1,00 \\
\hline Madre con secundaria completa & 11,734 & 0,28 & 0,45 & 0,00 & 1,00 \\
\hline Madre técnica/tecnológica incompleta & 11,734 & 0,01 & 0,11 & 0,00 & 1,00 \\
\hline Madre técnica/tecnológica completa & 11,73 & 0,05 & 0,21 & 0,00 & 1,00 \\
\hline Madre profesional incompleta & 11,734 & 0,01 & 0,10 & 0,00 & 1,00 \\
\hline Madre profesional completa & 1,734 & 0,04 & 0,21 & 0,00 & 1,00 \\
\hline Madre con posgrado & 11,734 & 0,01 & 0,10 & 0,00 & 1,00 \\
\hline
\end{tabular}




\begin{tabular}{|c|c|c|c|c|c|}
\hline Variable & Observ. & Media & Desv. Est. & Mínimo & Máximo \\
\hline Estrato 1 & 11,734 & 0,32 & 0,47 & 0,00 & 1,00 \\
\hline Estrato 2 & 11,734 & 0,44 & 0,50 & 0,00 & 1,00 \\
\hline Estrato 3 & 11,734 & 0,23 & 0,42 & 0,00 & 1,00 \\
\hline Estrato 4 & 11,734 & 0,01 & 0,10 & 0,00 & 1,00 \\
\hline Estrato 5 & 11,734 & 0,00 & 0,04 & 0,00 & 1,00 \\
\hline Estrato 6 & 11,734 & 0,00 & 0,02 & 0,00 & 1,00 \\
\hline \multicolumn{6}{|l|}{ Tipo de jornada académica (1=Sí) } \\
\hline Asiste jornada completa & 11,734 & 0,21 & 0,40 & 0.00 & 1,00 \\
\hline Asiste jornada mañana & 11,734 & 0,57 & 0,50 & 0.00 & 1,00 \\
\hline Asiste jornada tarde & 11,734 & 0,22 & 0,42 & 0.00 & 1,00 \\
\hline \multicolumn{6}{|l|}{ Establecimiento } \\
\hline $\begin{array}{l}\text { Tiempo promedio efectivo de instrucción } \\
(\%)\end{array}$ & 11,734 & 65,42 & 17,27 & 0,00 & 100,00 \\
\hline Edad promedio de los estudiantes & 11,734 & 16,45 & 0,36 & 15,04 & 19,06 \\
\hline Proporción estudiantes nuevos & 11,734 & 0,04 & 0,05 & 0,00 & 0,47 \\
\hline Proporción estudiantes cabeza de familia & 11,734 & 0,00 & 0,02 & 0,00 & 0,22 \\
\hline Proporción estudiantes repitentes & 11,734 & 0,04 & 0,05 & 0,00 & 0,34 \\
\hline Número de estudiantes en grado 11 & 11,734 & 104,39 & 63,81 & 6,00 & 282 \\
\hline $\begin{array}{l}\text { Promedio del colegio en Matemáticas en } \\
\text { Saber } 11 \text { de } 2010\end{array}$ & 11,734 & 49,31 & 3,54 & 39,57 & 60,72 \\
\hline $\begin{array}{l}\text { Varianza del colegio en Matemáticas en } \\
\text { Saber } 11 \text { de } 2010\end{array}$ & 11,734 & 77,23 & 19,04 & 22,69 & 174,96 \\
\hline $\begin{array}{l}\text { Promedio del colegio en Lenguaje en Sa- } \\
\text { ber } 11 \text { de } 2010\end{array}$ & 11,734 & 49,80 & 3,03 & 42,40 & 60,12 \\
\hline $\begin{array}{l}\text { Varianza del colegio en Lenguaje en Saber } \\
11 \text { de } 2010\end{array}$ & 11,734 & 81,10 & 18,87 & 31,43 & 158,30 \\
\hline Edad promedio de los docentes & 11,734 & 44,02 & 4,28 & 29,00 & 54,14 \\
\hline
\end{tabular}

Fuente: cálculos del autor con datos del Banco Mundial, ICFEs y MEN. 


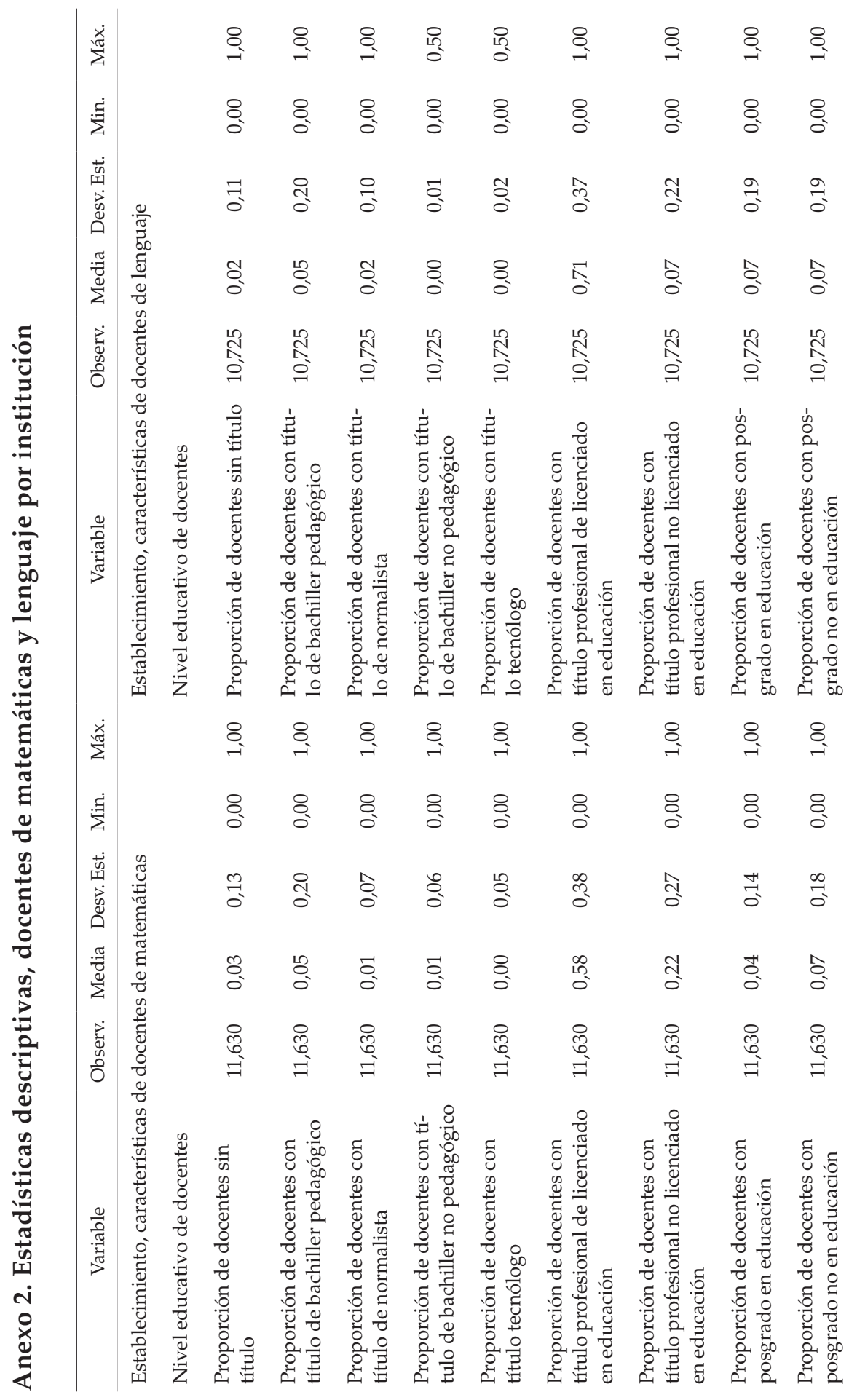




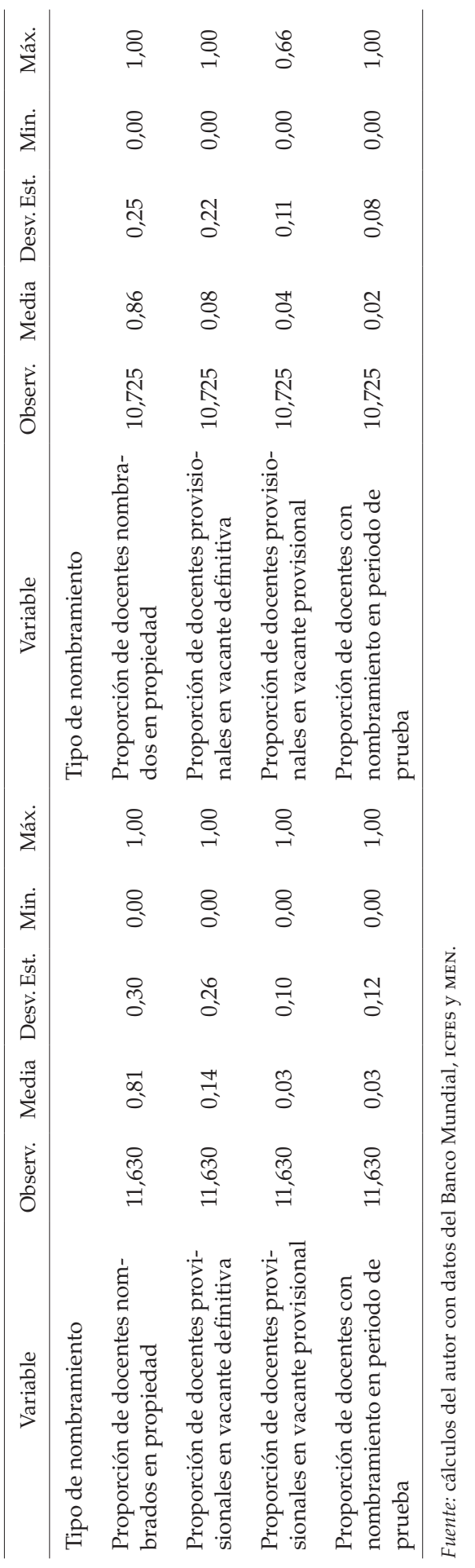




\section{Anexo 3. Resultados prueba para detectar endogeneidad de Hausman}

\begin{tabular}{lcc}
\hline & Matemáticas & Lenguaje \\
\hline Residuales Predichos en Primera Etapa ${ }^{16}$ & $-0,390^{* * *}$ & $-0,111$ \\
Características de docentes & $(0,115)$ & $(0,095)$ \\
Efectos fijos de municipio & Sí & Sí \\
Características de establecimiento & Sí & Sí \\
Características de estudiante y familia & Sí & Sí \\
Observaciones & Sí & Sí \\
R-cuadrado & 11,630 & 10,725 \\
\hline
\end{tabular}

Errores estándar robustos en paréntesis ${ }^{* * *} \mathrm{p}<0,01,{ }^{* *} \mathrm{p}<0,05,{ }^{*} \mathrm{p}<0,1$

Fuente: cálculos del autor con datos del Banco Mundial, ICFES y MEN.

\section{Anexo 4. Resultados prueba para restricciones sobreidentificadas de Sargan}

\begin{tabular}{lcc}
\hline & Residuales Matemáticas & Residuales Lenguaje \\
\hline Edad promedio de los docentes al cuadrado & 0,003 & 0,008 \\
Características de docentes & $(0.023)$ & $(0.025)$ \\
Efectos fijos de municipio & Sí & Sí \\
Características de establecimiento & Sí & Sí \\
Características de estudiante y familia & Sí & Sí \\
Observaciones & Sí & 10,725 \\
R-cuadrado & 11,630 & 0,000 \\
\hline
\end{tabular}

Errores estándar robustos en paréntesis ${ }^{* * *} \mathrm{p}<0,01,{ }^{* *} \mathrm{p}<0,05,{ }^{*} \mathrm{p}<0,1$

Fuente: cálculos del autor con datos del Banco Mundial, ICFES y MEN.

16 Ver tabla 2. 


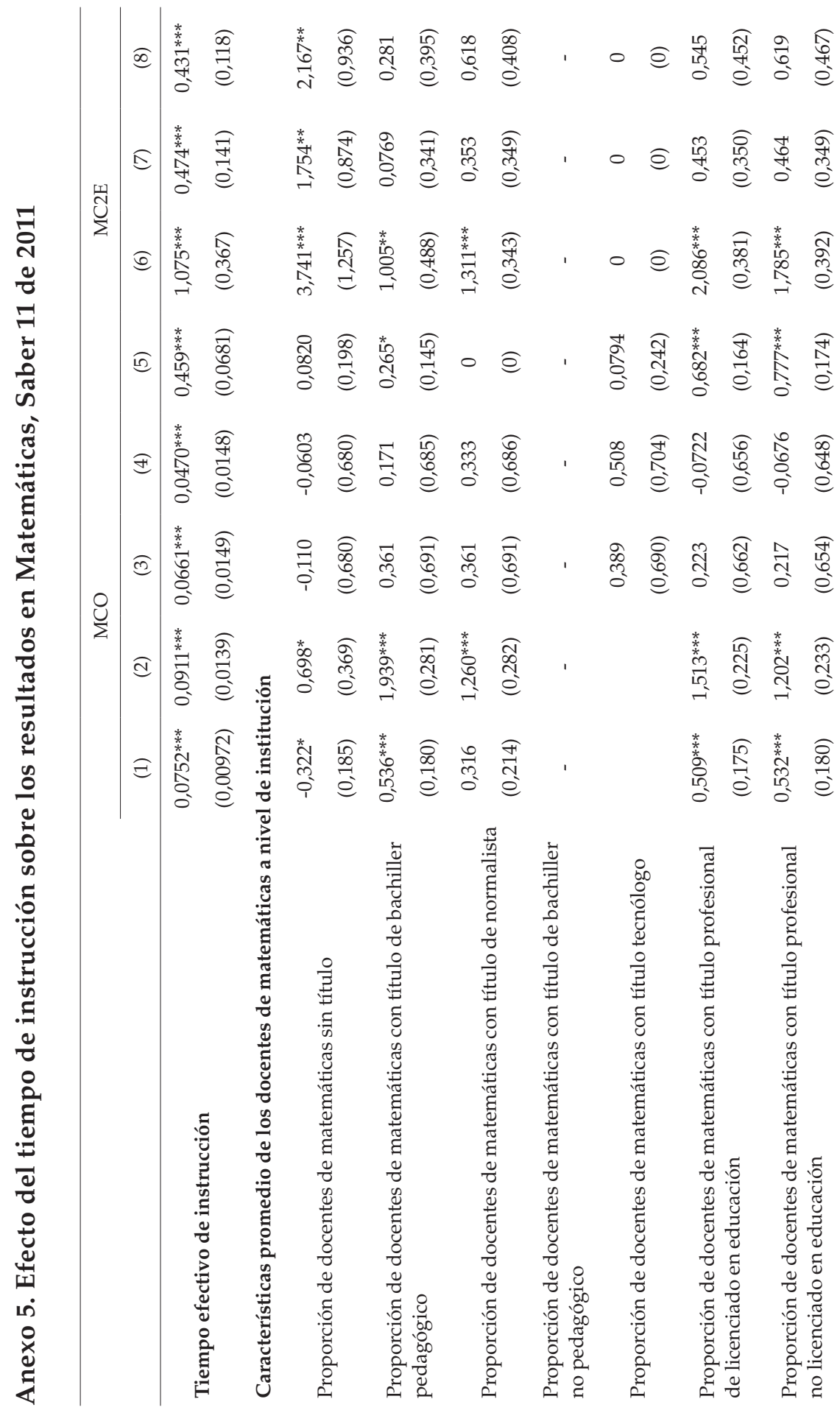




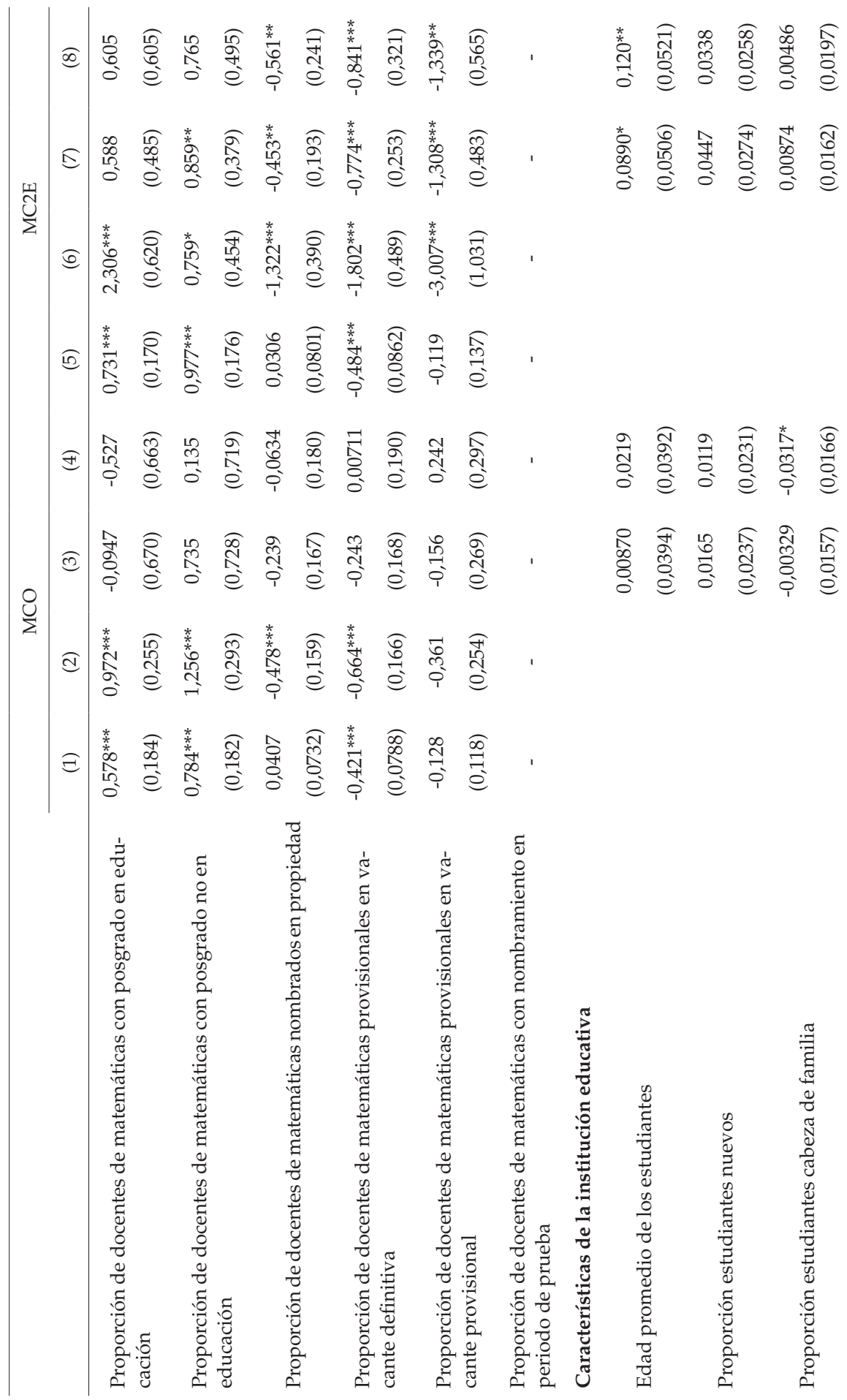




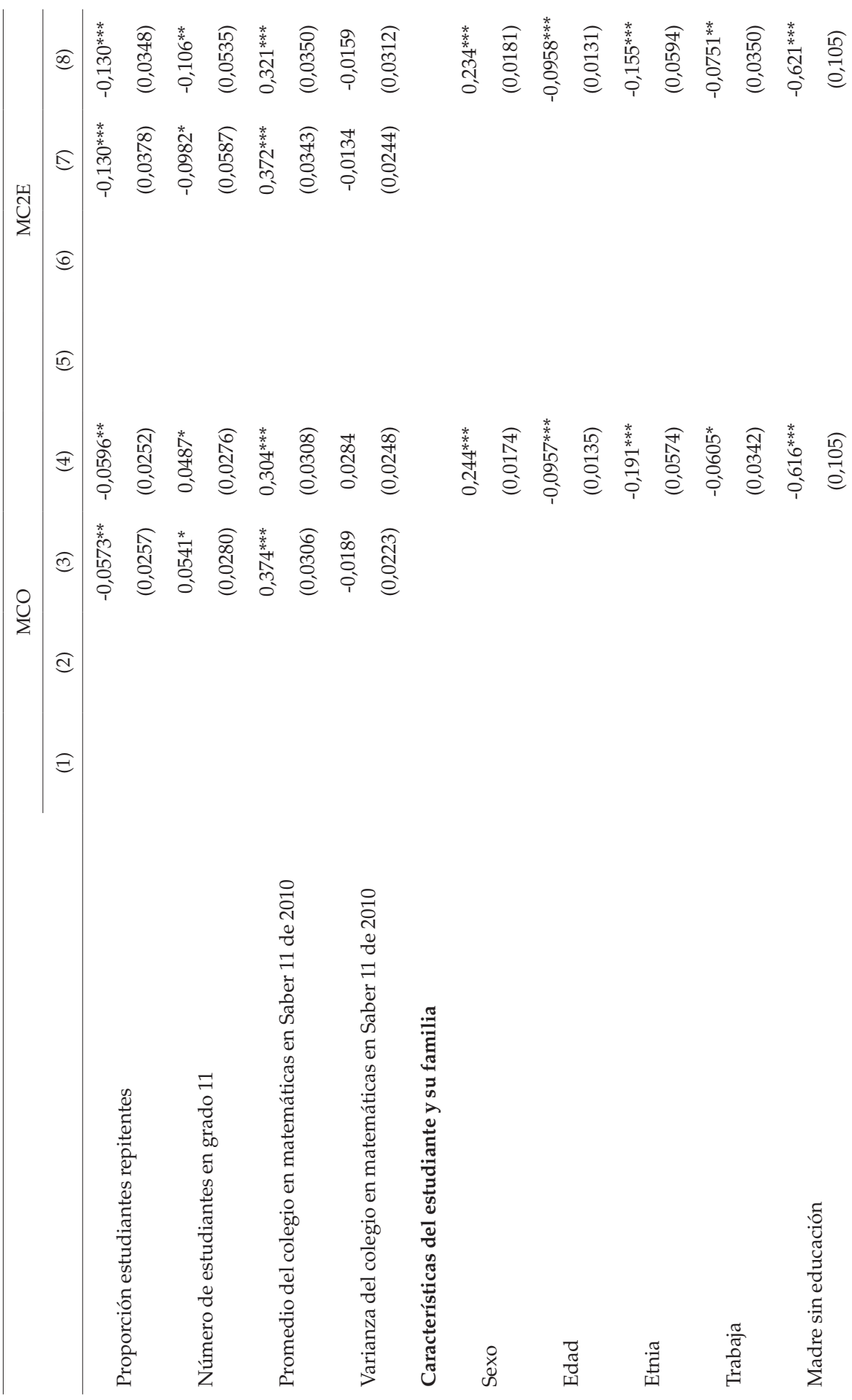




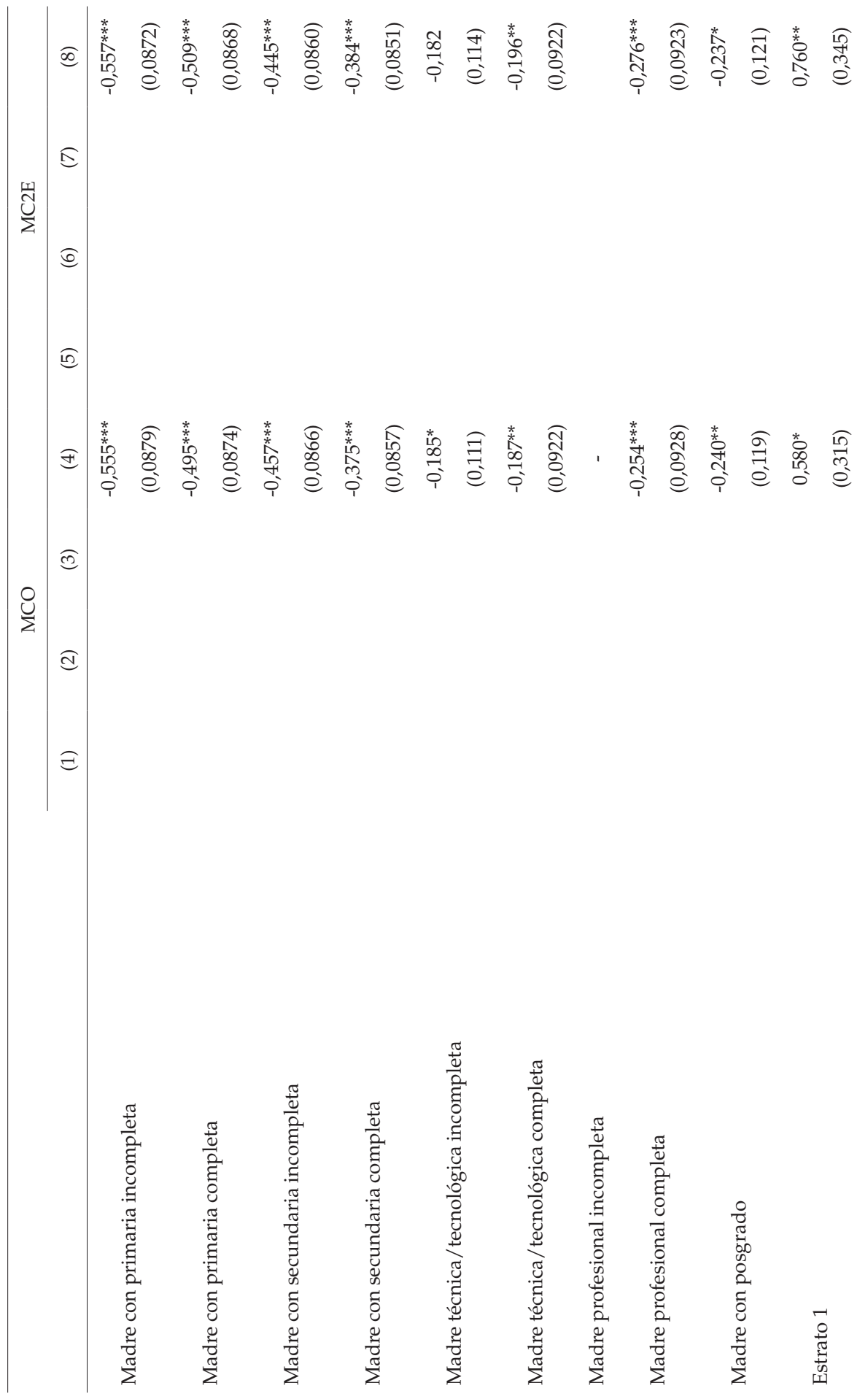




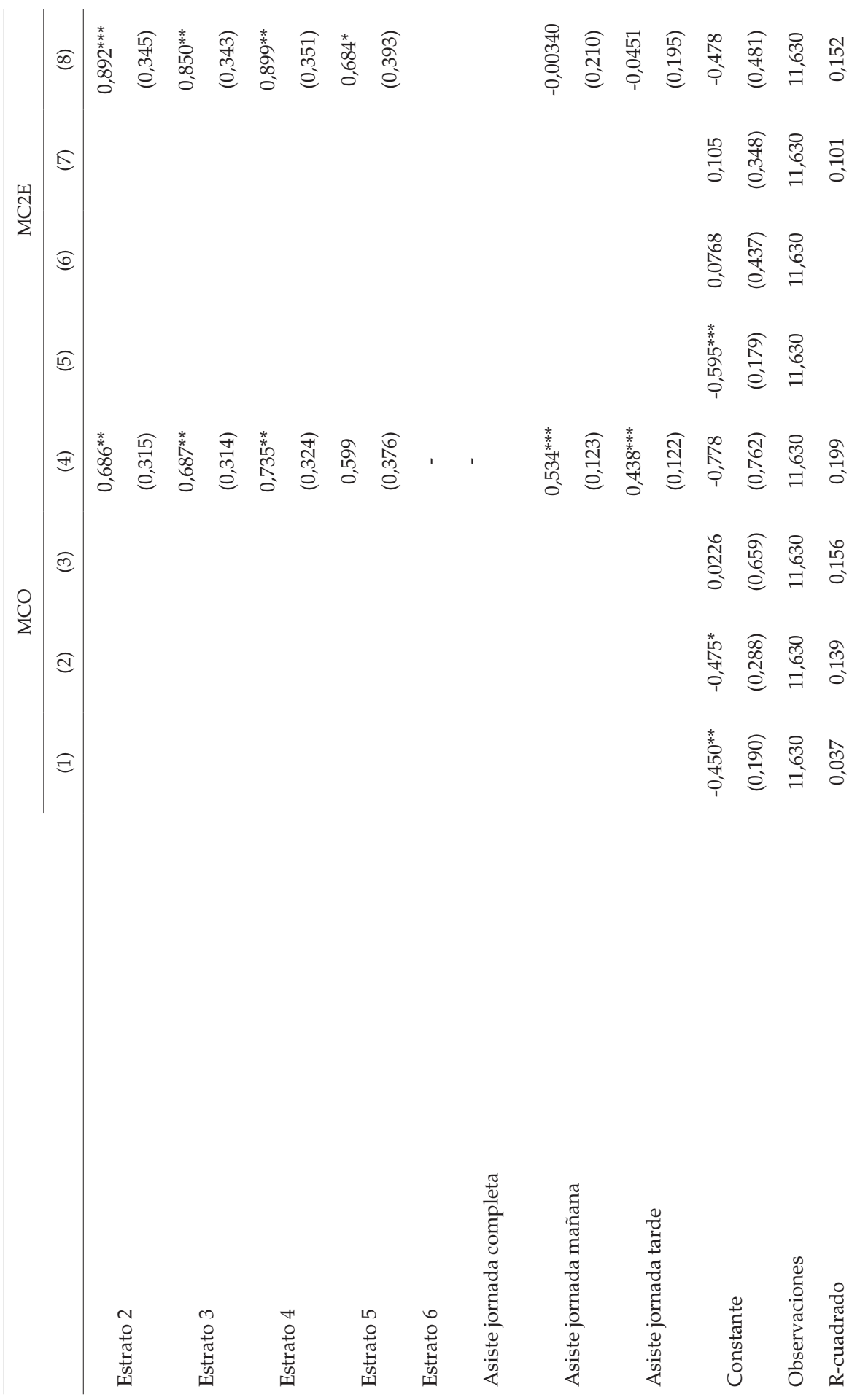




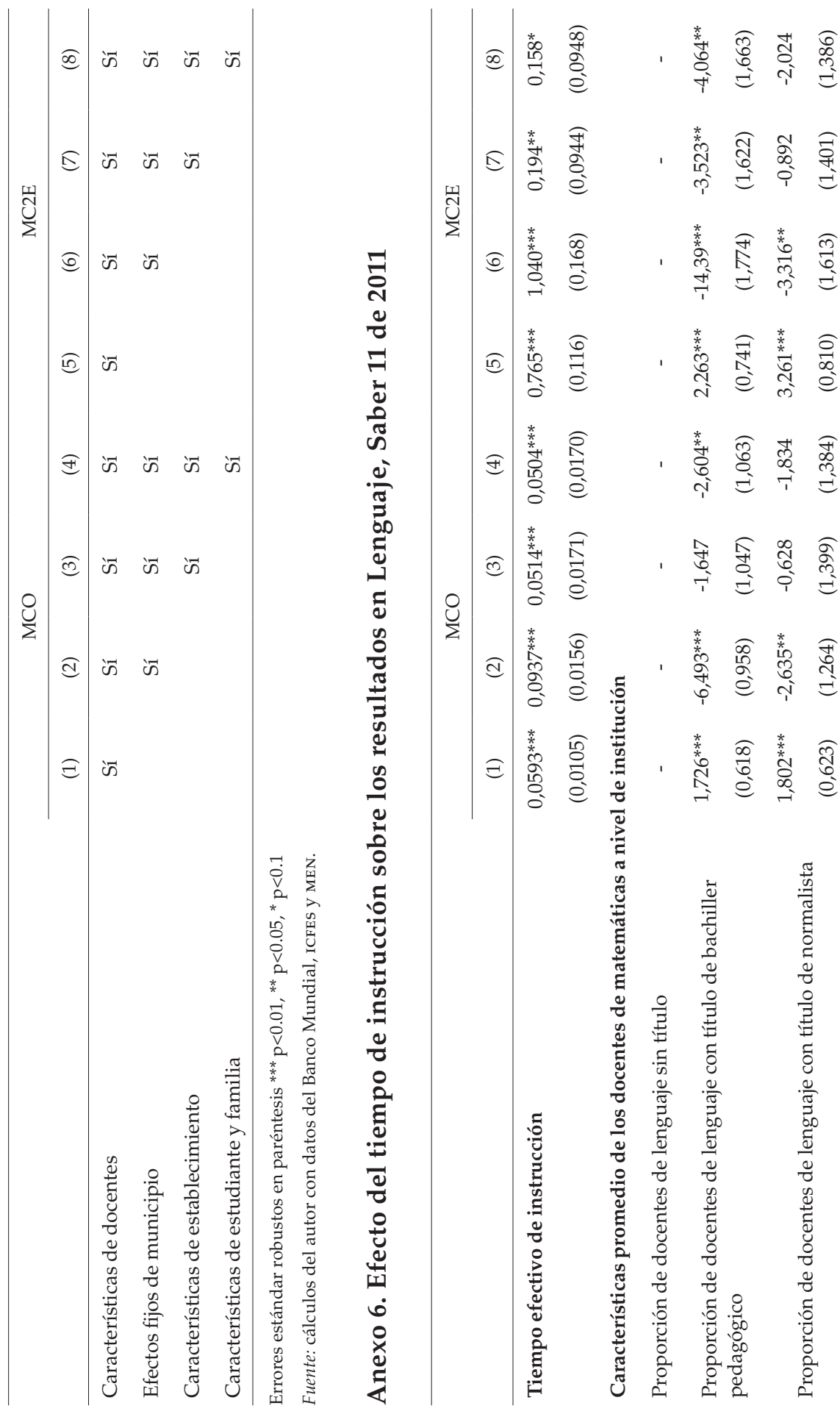




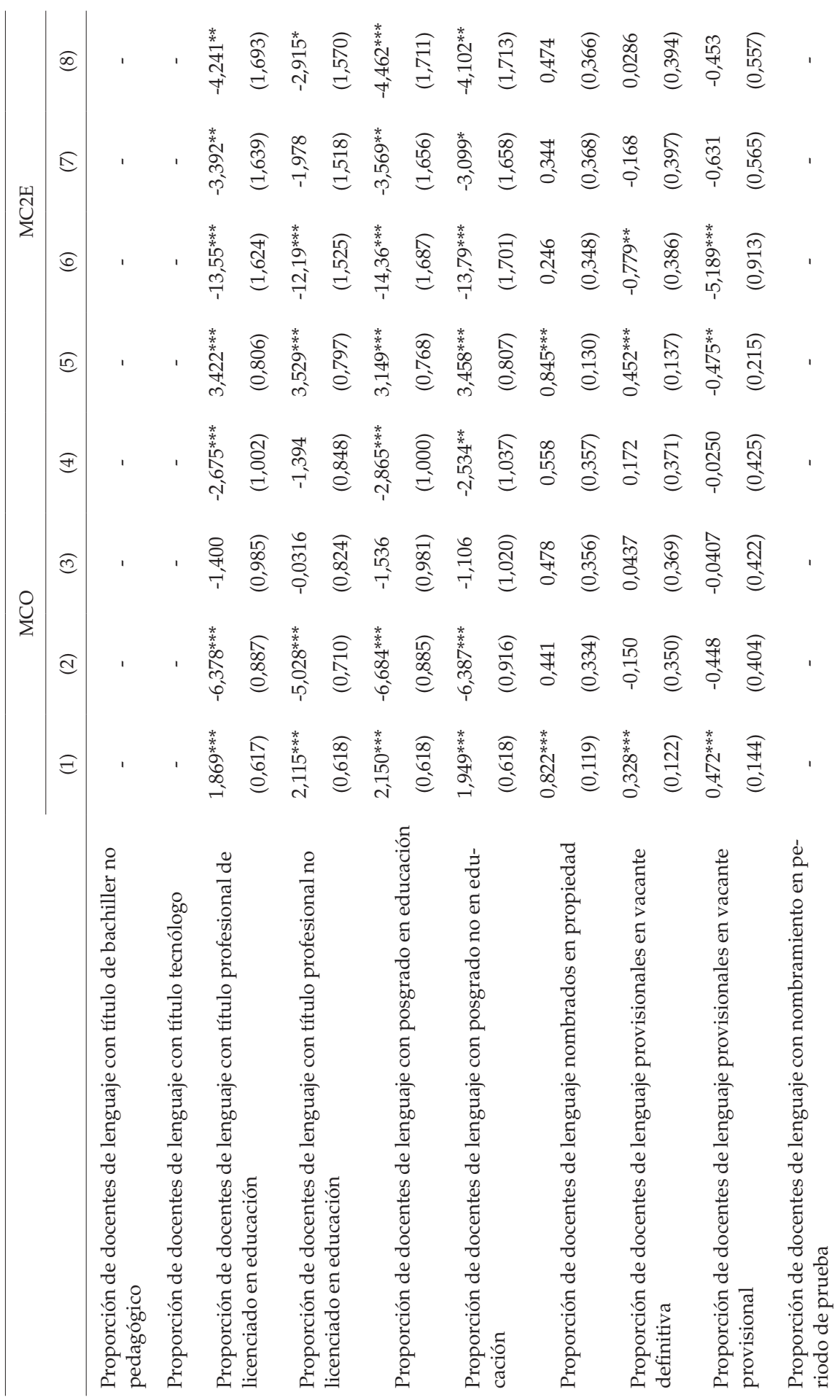




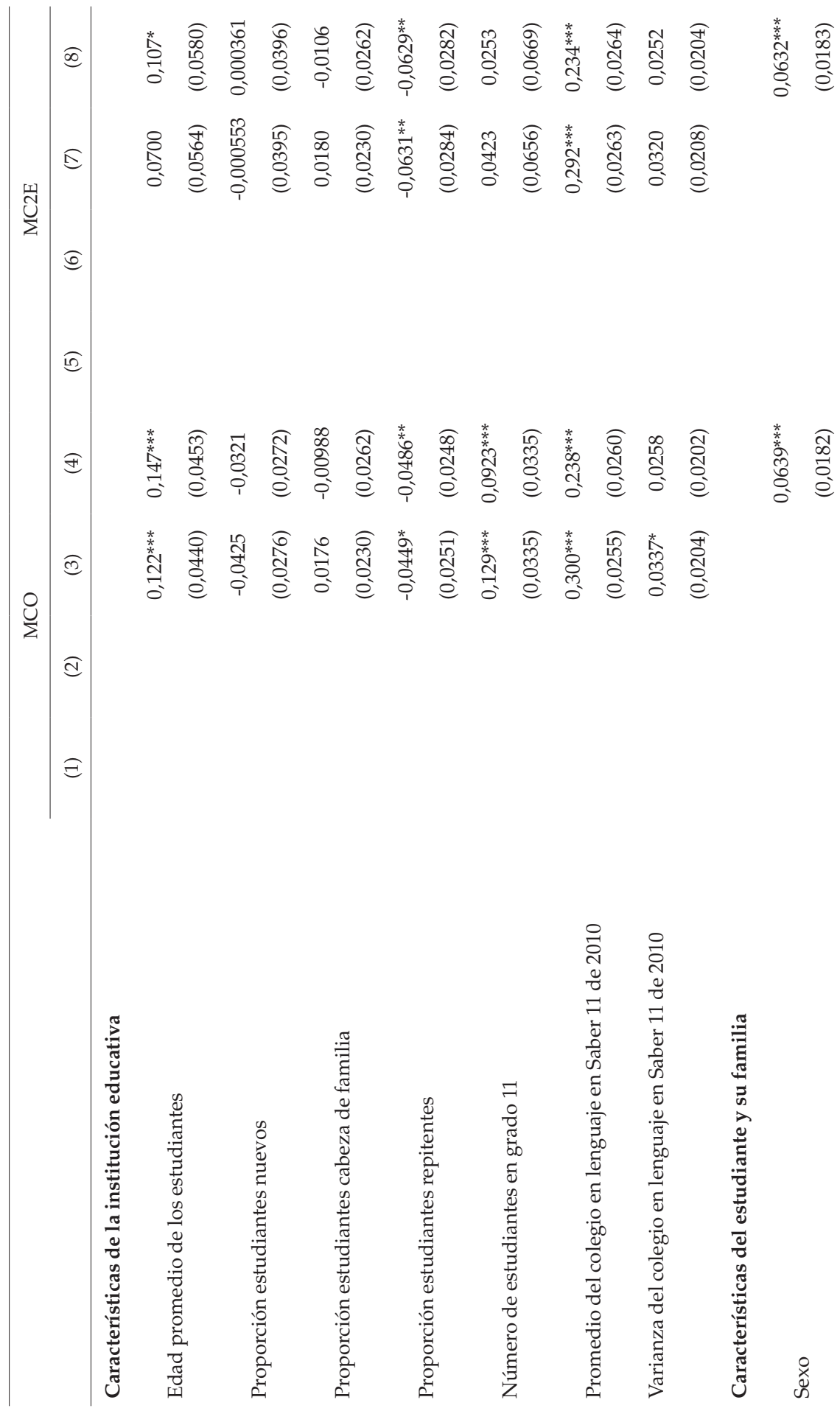




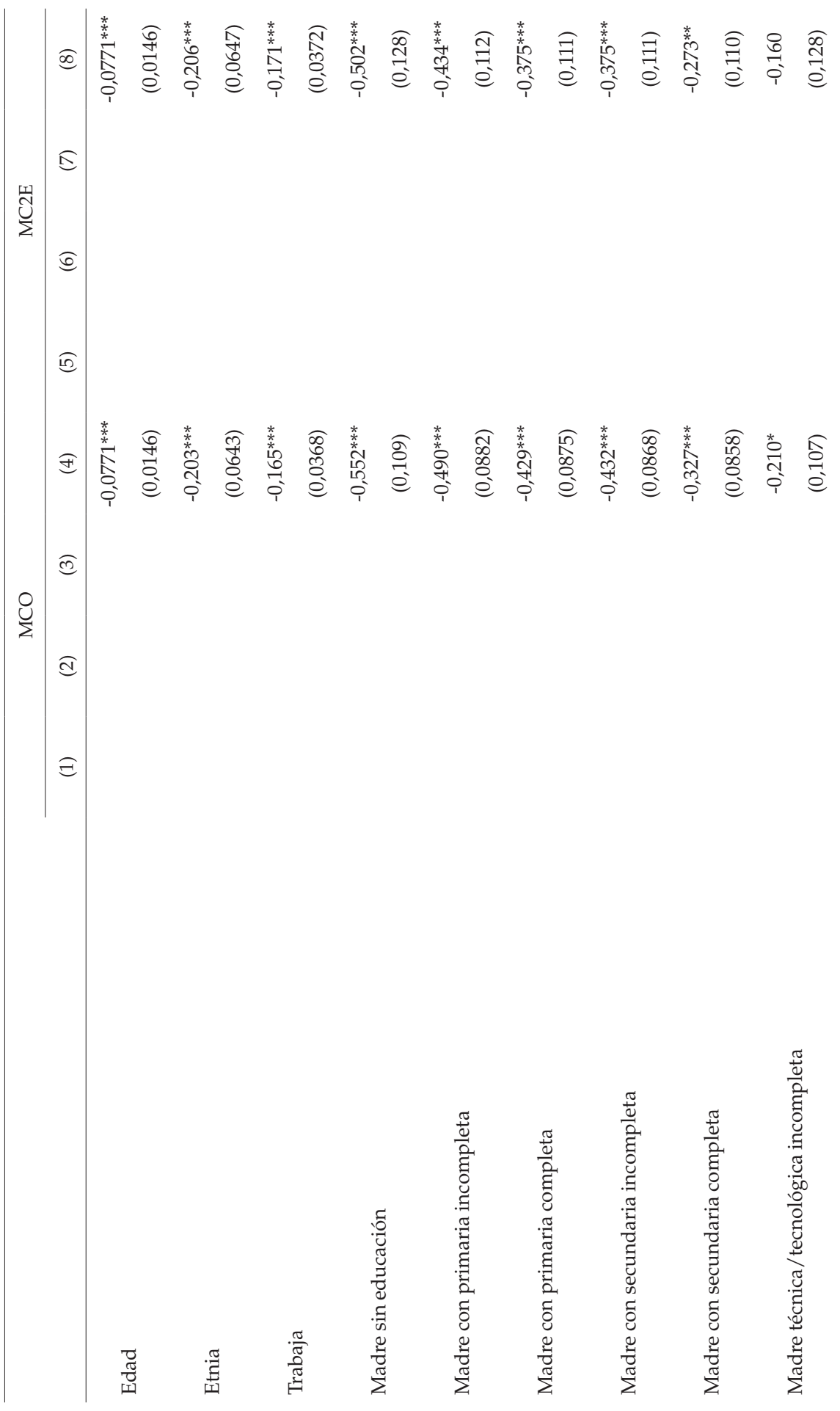




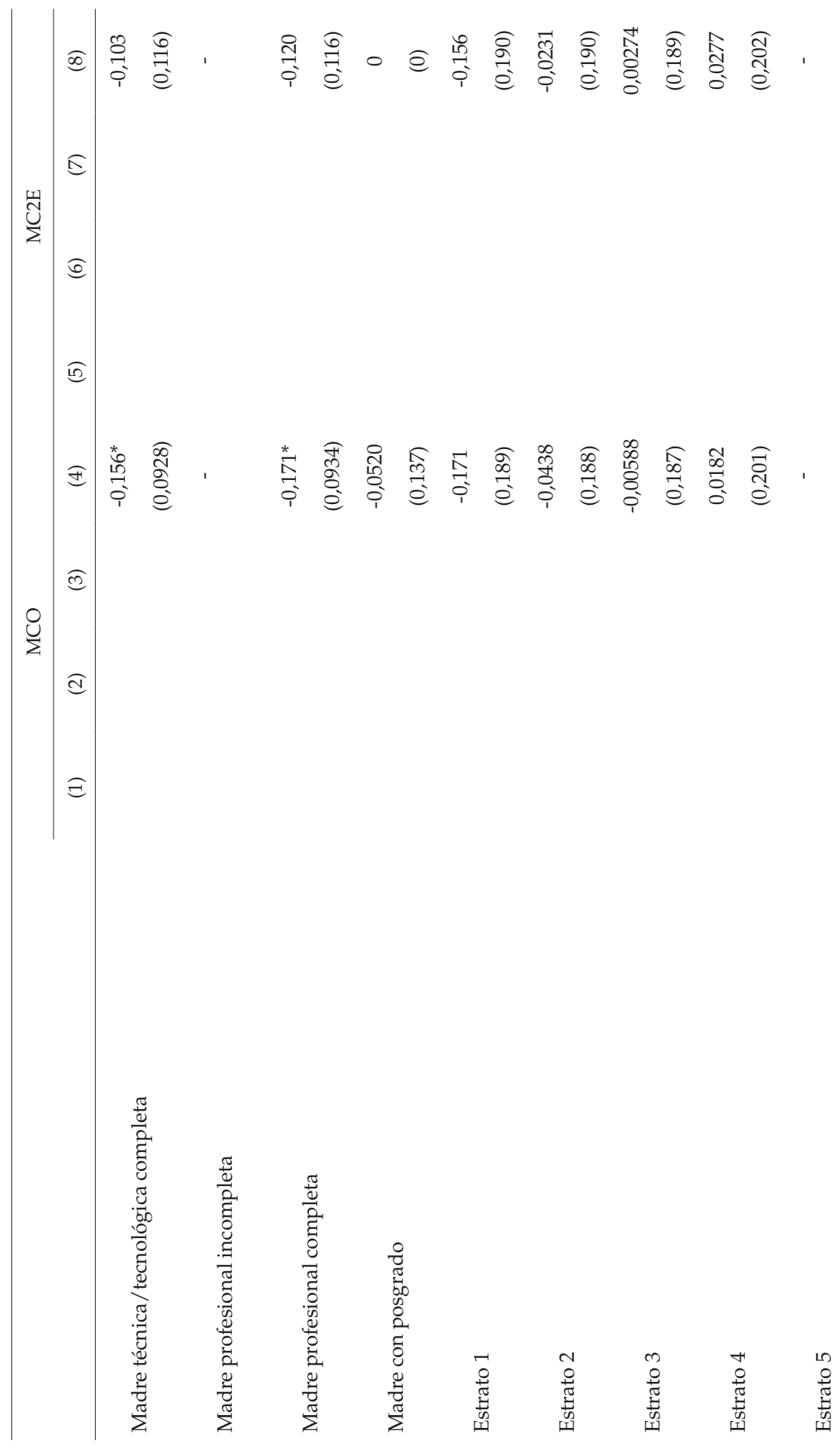




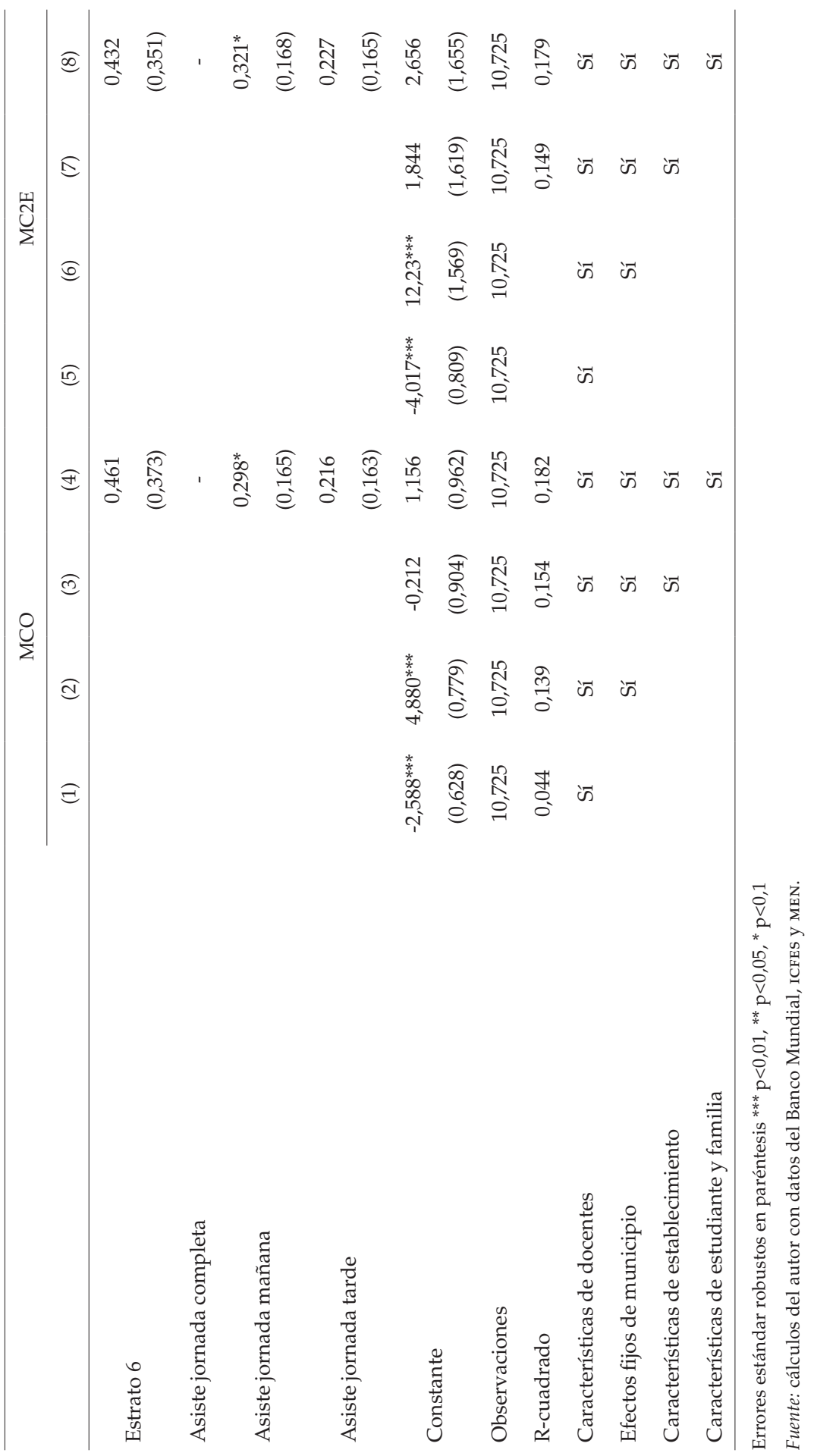




\section{Anexo 7. Formato de entrevista semiestructurada}

\section{Formato de entrevista semiestructurada}

\section{Tesis "Desempeño escolar y tiempo efectivo de instrucción en Colombia"}

\section{Objetivo:}

- Indagar sobre la utilización del tiempo de clase y sus consecuencias.

- Consultar los principales obstáculos y/o facilitadores al normal desarrollo de las clases.

\section{Participantes:}

- Ocho docentes del departamento del Atlántico, cuatro de lenguaje y cuatro de matemáticas en los grados 10 u 11.

\section{Actividades:}

Primer paso: agradecer el tiempo prestado para la entrevista.

Segundo paso: confirmar consentimiento informado a los participantes (es importante dejar registro en el audio del consentimiento).

Buenos días, mi nombre es y soy el autor de esta investigación, me encuentro con [nombre del participante] y le leeré el consentimiento informado para participar en el estudio.

El objetivo de su participación en el estudio es incluir su opinión en una investigación sobre el uso del tiempo de clase en Colombia. La información que usted brinde durante este proceso contribuirá a la construcción de una publicación que estimará la influencia del uso del tiempo de instrucción en el desempeño de los estudiantes colombianos y visibilizará las experiencias de docentes alrededor de esta relación. Este proyecto de investigación es requisito de grado del doble programa entre la Maestría en Políticas Públicas y la Maestría en Economía de la Universidad de los Andes.

Descripción de actividades: si usted decide colaborar con este proyecto, participará en actividades de recolección de datos cualitativos: contestar preguntas generales sobre su vida diaria en la escuela, su uso del tiempo de clase, sobre factores que piense que afectan el normal desarrollo de las actividades 
educativas y sobre propuestas, propias o de su comunidad educativa, que busquen mejorar el uso del tiempo de clase. Usted no tiene que responder a todas las preguntas.

Beneficios: este estudio no está diseñado para ayudarlo(a) a usted directamente, pero lo aquí aprendido puede ayudar a otras personas en el futuro y aportar a la elaboración de sugerencias de política pública en educación.

Confidencialidad: toda la información que usted comparta en este estudio es confidencial. En ningún momento se revelará su nombre o identidad, así como tampoco se revelarán los datos individuales, pues estos serán usados de manera anónima en escritos académicos exclusivamente por mi persona.

Compensación: por las actividades de recolección de datos (entrevistas) no recibirá una compensación monetaria.

Participación voluntaria: usted no está obligado a participar en el estudio. Si usted decide no participar o si decide retirarse del estudio en cualquier momento, esto no le generará ningún perjuicio. Aunque decida participar o no, su relación con el investigador no se verá afectada por este estudio.

Si tiene alguna pregunta durante o después de que termine el estudio, usted puede escribir al correo electrónico XXXXXXXX@uniandes.edu.co. En caso de presentarse algún problema asociado a la investigación, usted también puede contactar al Comité de Ética de la Universidad de los Andes. Teléfono 3394949, Ext. 2073 o al correo electrónico comite_de_etica_egob@uniandes. edu.co

¿Quisiera usted participar en el estudio? [Respuesta del participante]

¿Podemos grabar la entrevista en audio para facilitar su procesamiento y análisis? [Respuesta del participante]

\section{Eje de conversación 1: caracterización del entrevistado (5 minutos)}

1.1. Nombre, asignaturas dictadas, ¿trabaja en otra escuela?.

1.2. ¿Cuál es su máximo grado educativo alcanzado?

1.3. ¿Estudió una carrera de formación docente (Licenciatura)? ¿Qué estudió?

1.4. ¿Desde cuándo se dedica a la docencia? 
2. Eje de conversación 2: uso del tiempo de clase (20 minutos)

2.1. ¿Cree que las horas de clase son suficientes para cubrir el currículo de $\mathrm{su}(\mathrm{s})$ asignatura(s)?

2.2. ¿El uso del tiempo de clase es un asunto relevante para usted?

2.2.1. ¿Y para su comunidad educativa?

2.3. ¿En promedio, qué porcentaje de la clase diría que dedica a actividades académicas?

2.4. ¿Qué elementos asociados a su persona impiden que dedique el $100 \%$ de la clase a actividades académicas?

2.4.1. ¿Qué elementos identifica en sus estudiantes?

2.4.2. ¿Qué elementos identifica en las condiciones del aula o la escuela?

2.4.3. ¿Qué elementos identifica en la comunidad educativa?

2.5. ¿Qué factores identifica en su persona que lo incentiven o permitan aprovechar el tiempo total de clase?

2.5.1. ¿Qué factores identifica en sus estudiantes?

2.5.2. ¿Qué factores identifica en las condiciones del aula o la escuela?

2.5.3. ¿Qué factores identifica en la comunidad educativa?

3. Eje de conversación 3: consecuencias (10 minutos)

3.1. Desde su perspectiva, ¿cree que el uso inadecuado del tiempo de clase puede afectar el desempeño de sus estudiantes?

3.1.1. ¿Cómo cree que puede afectarlo?

4. Eje de conversación 4: propuestas de mejora (20 minutos)

4.1. ¿Cómo reduce la pérdida de tiempo en la clase?

4.2. ¿Ha propuesto algún tipo de iniciativas para mejorar el uso del tiempo de clase?

4.2.1. ¿Cuáles?

4.3. ¿Cree que la comunidad educativa es o podría ser receptiva a propuestas que busquen mejorar el uso del tiempo de clase?

4.4. ¿Desde la escuela se han propuesto algunas iniciativas al respecto?

4.4.1. ¿Cuáles?

4.4.2. ¿Se está(n) desarrollando actualmente?

5. Cierre de la entrevista

5.1. ¿Tiene algún comentario o sugerencia sobre lo conversado?

5.2. Agradecimientos. 
